
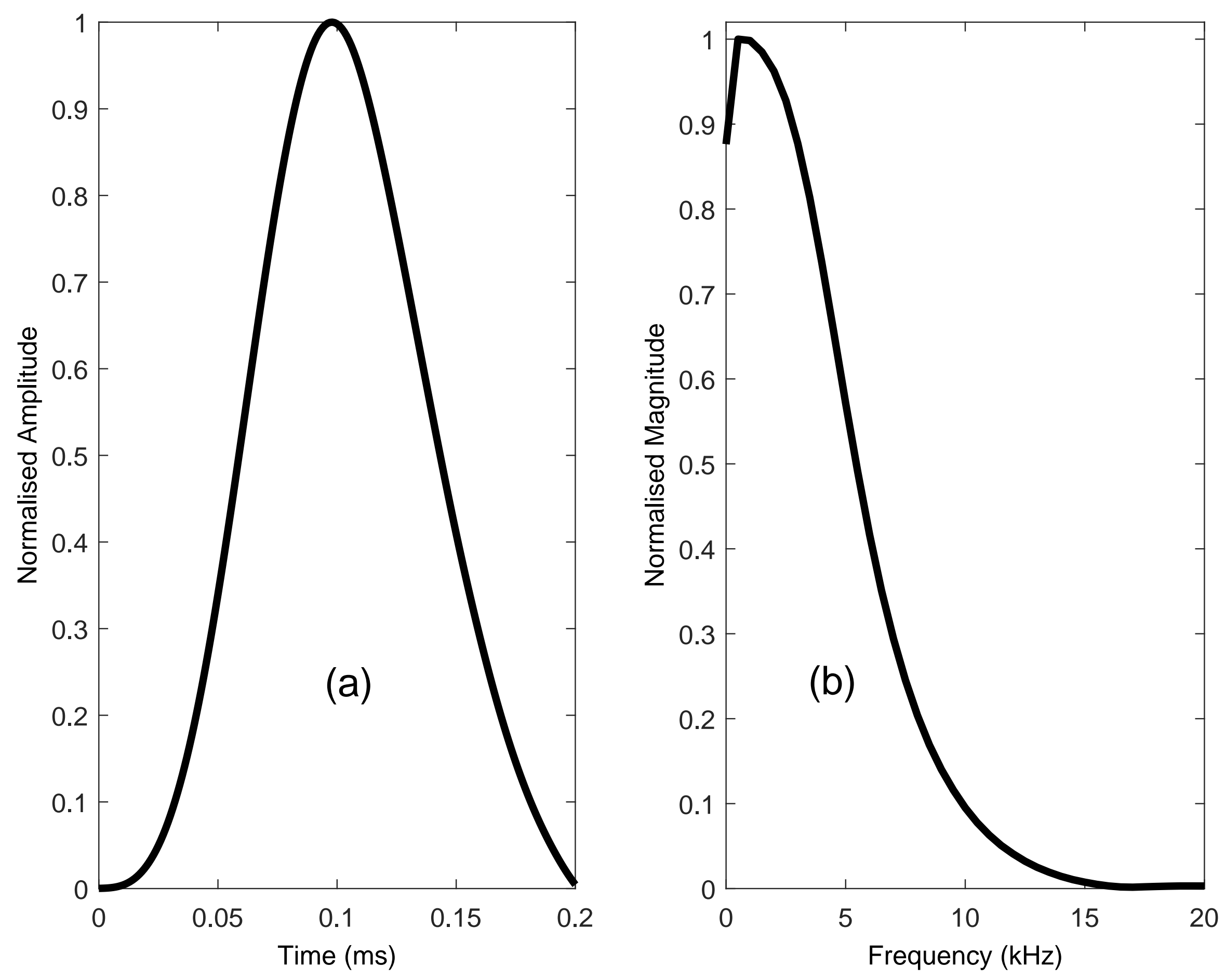


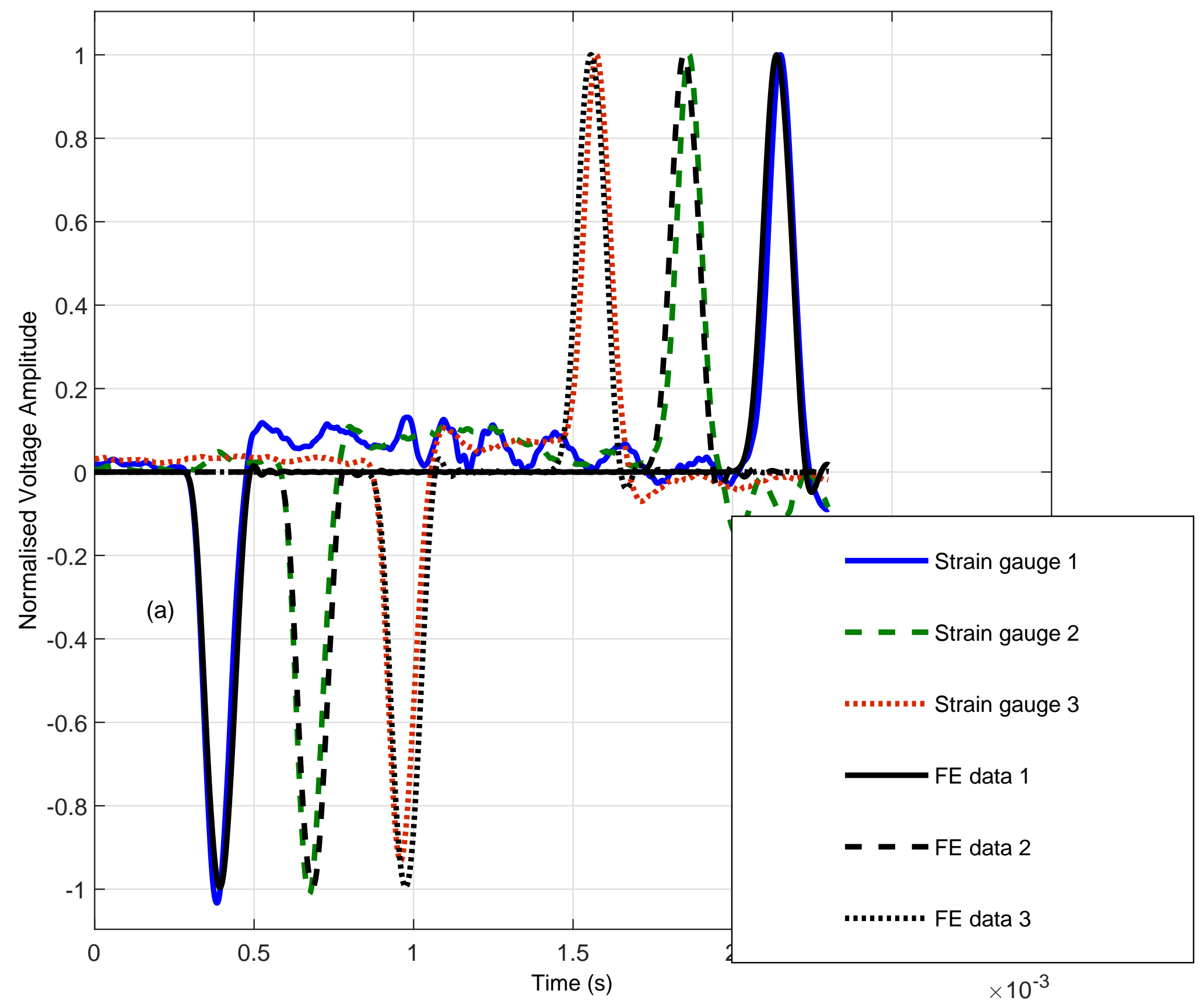


Comparison of FE with test data (a) time domain signals and (b) corresponding frequency spectrum

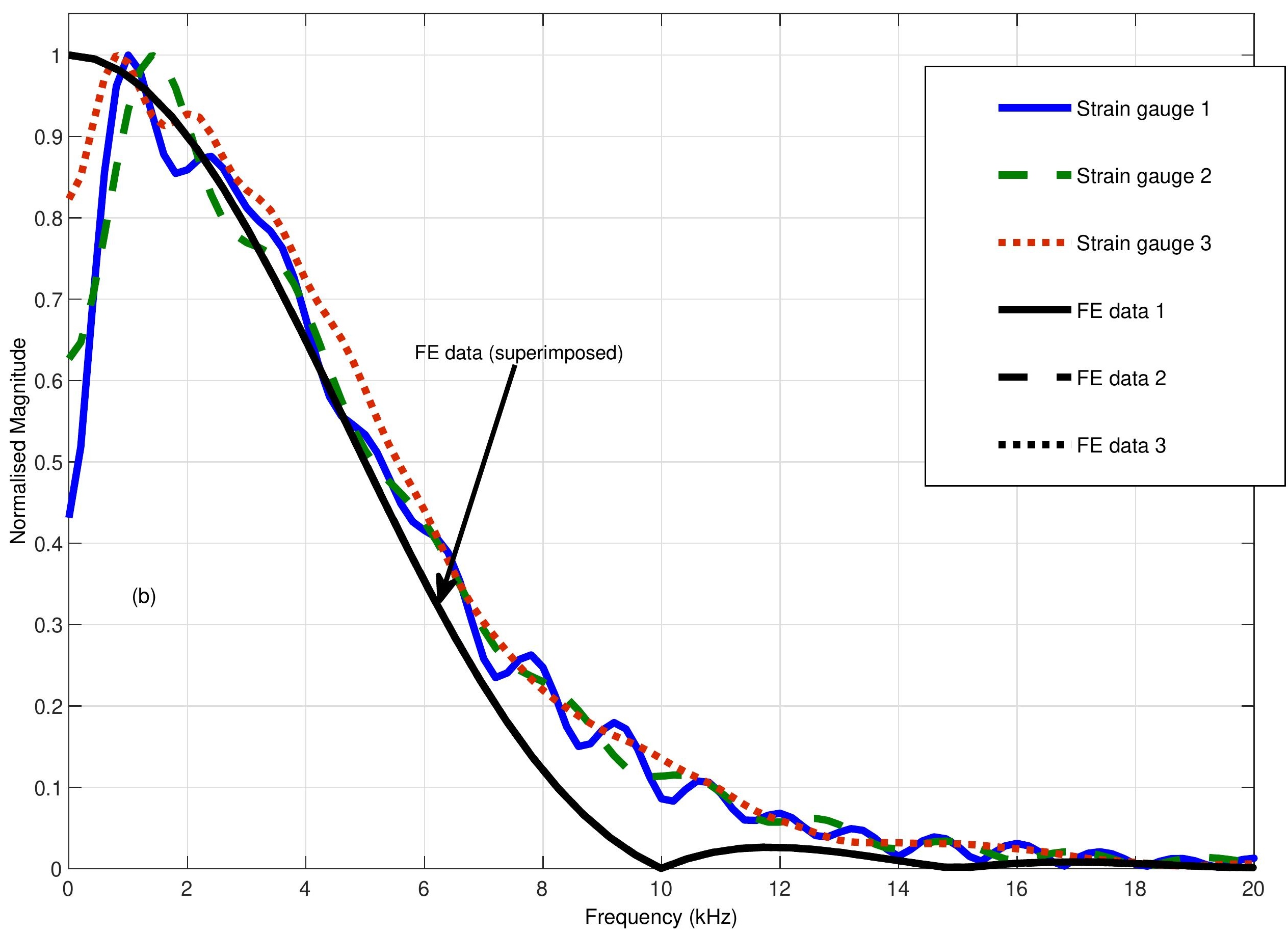



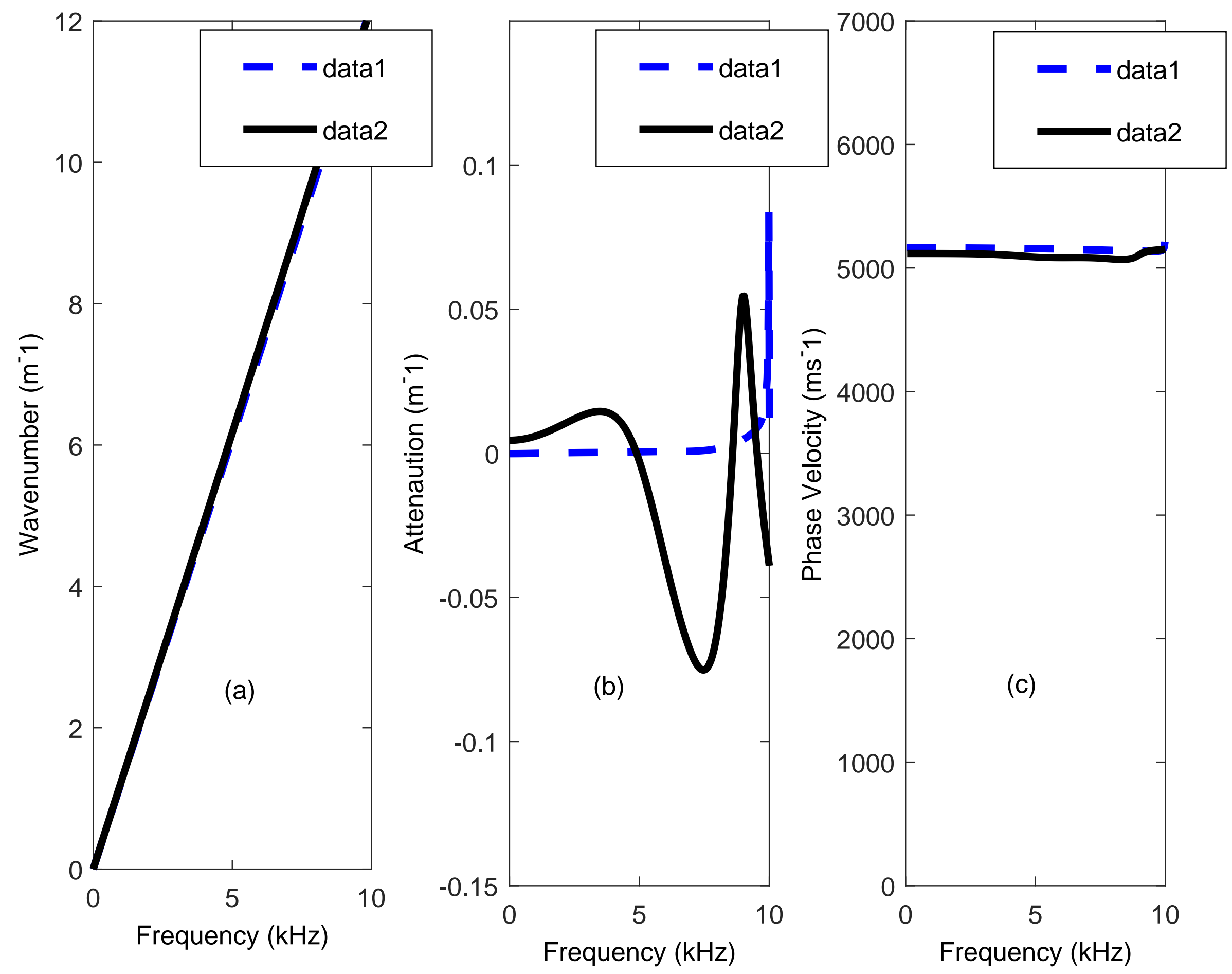


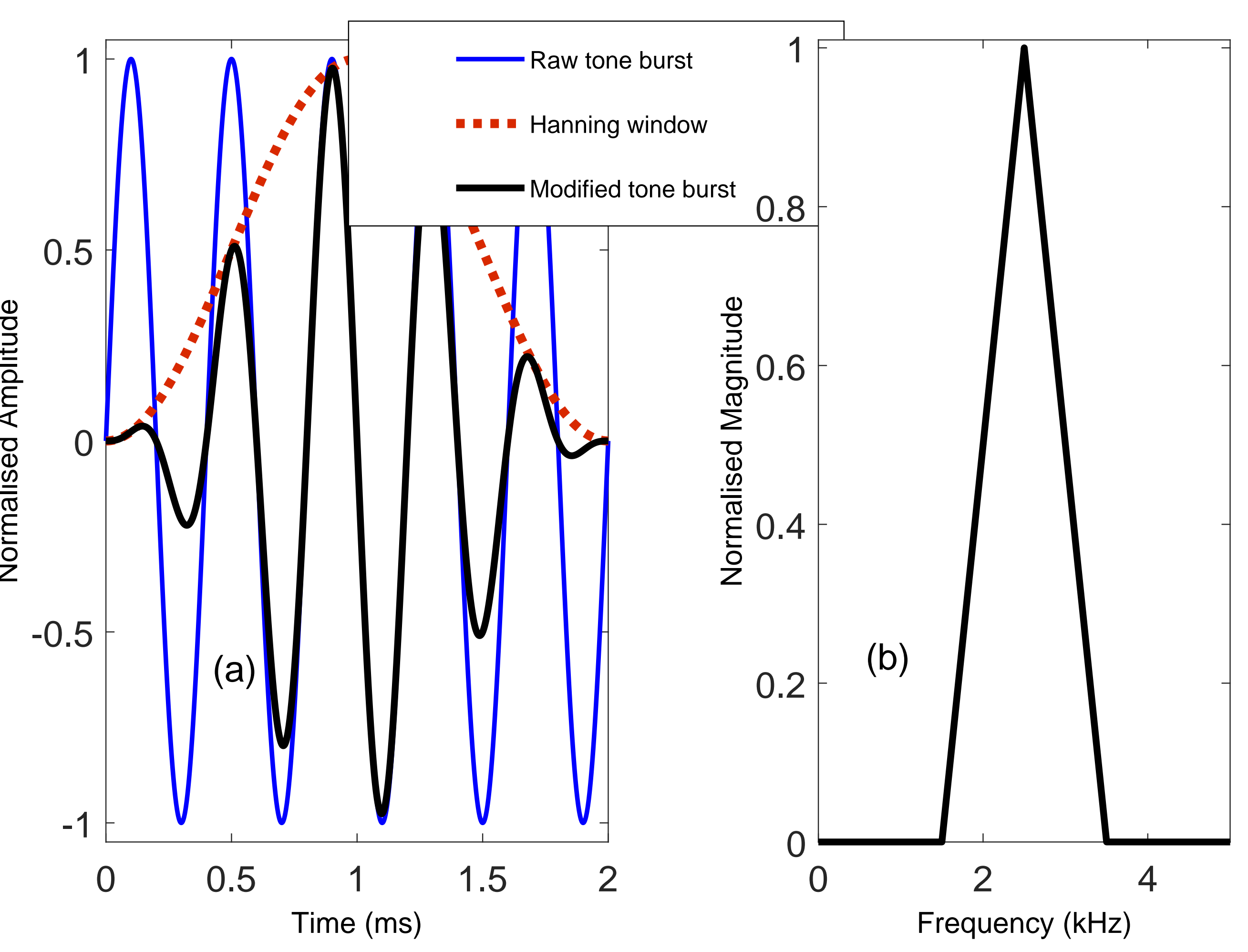



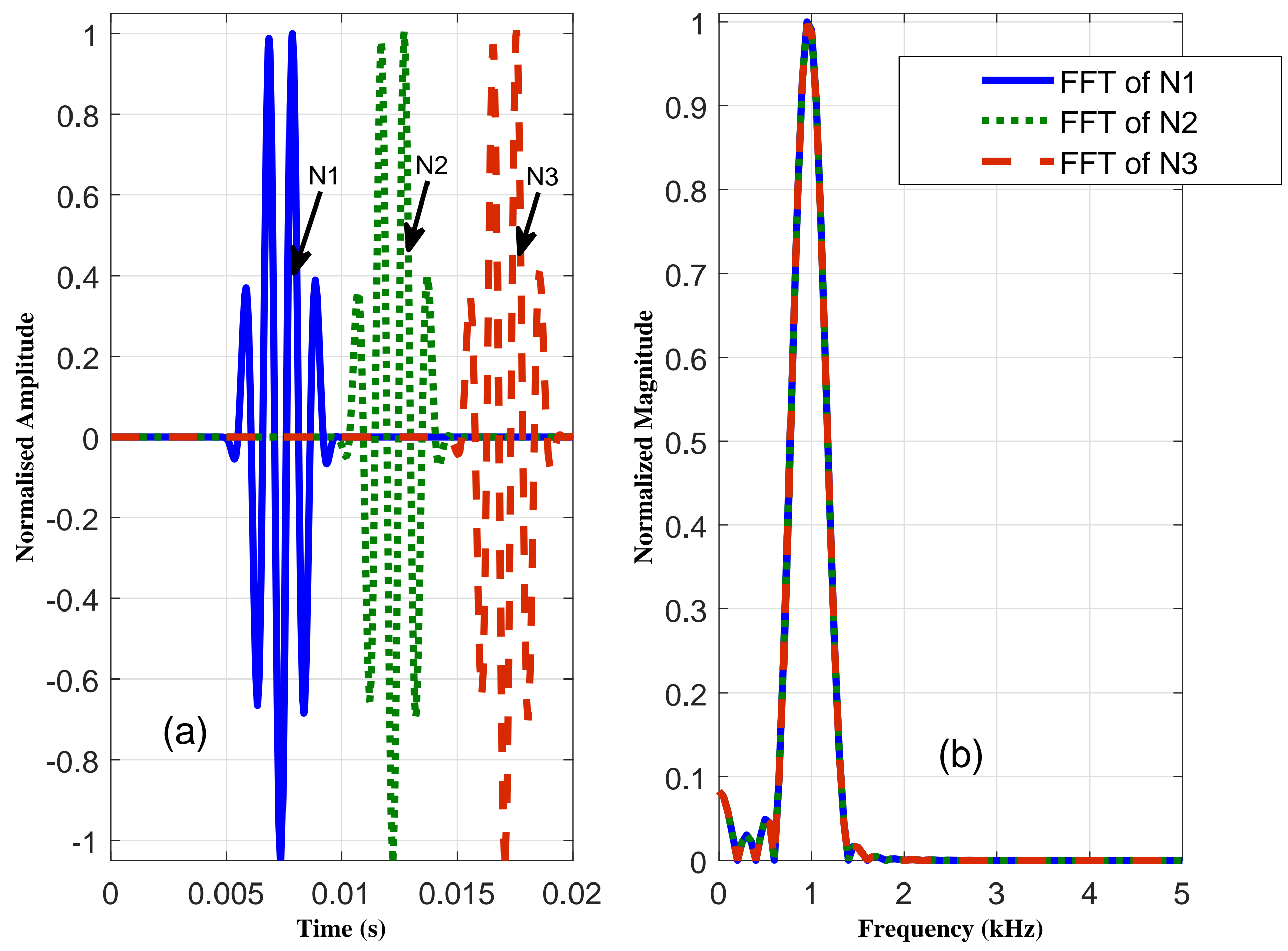

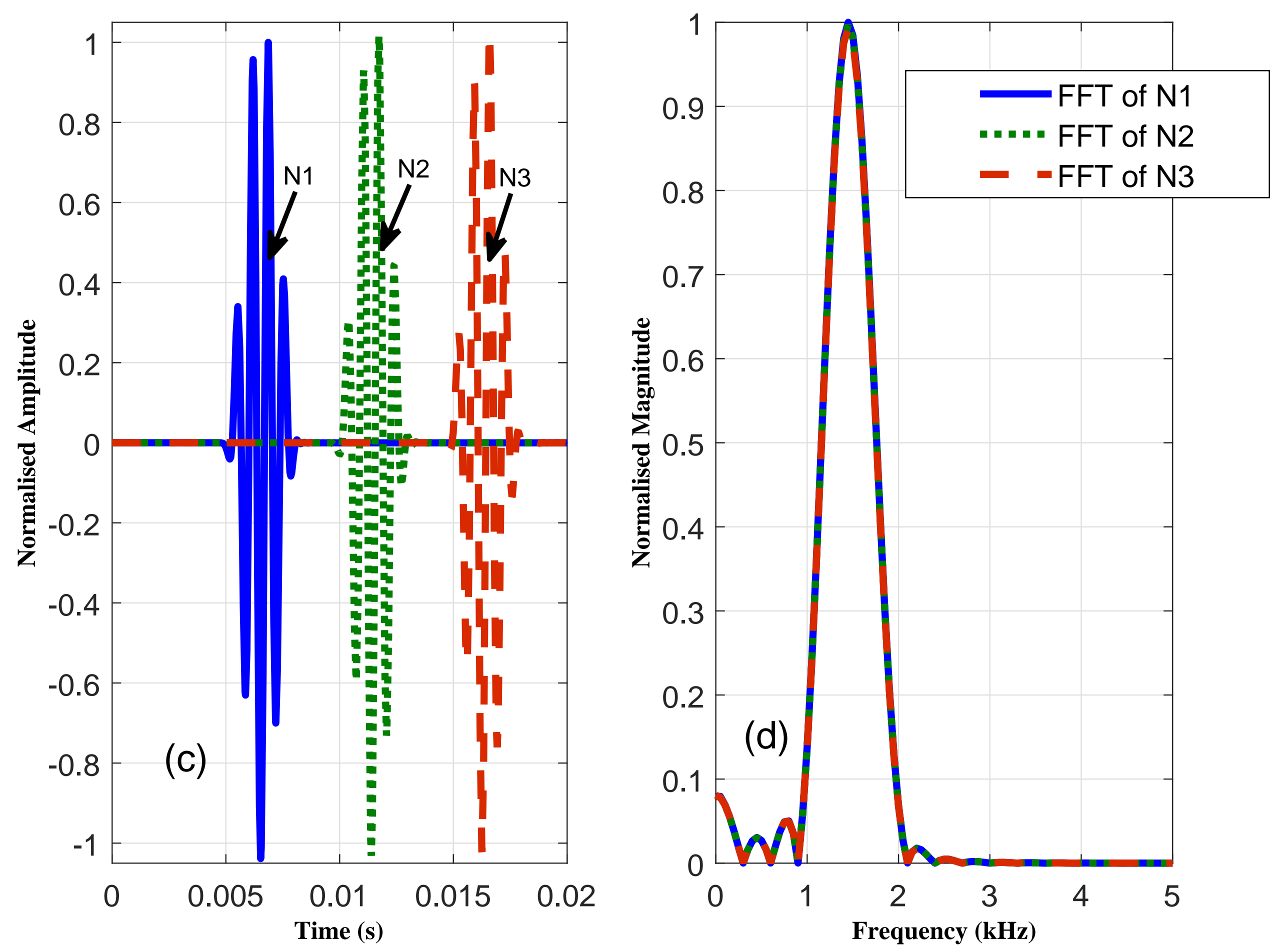

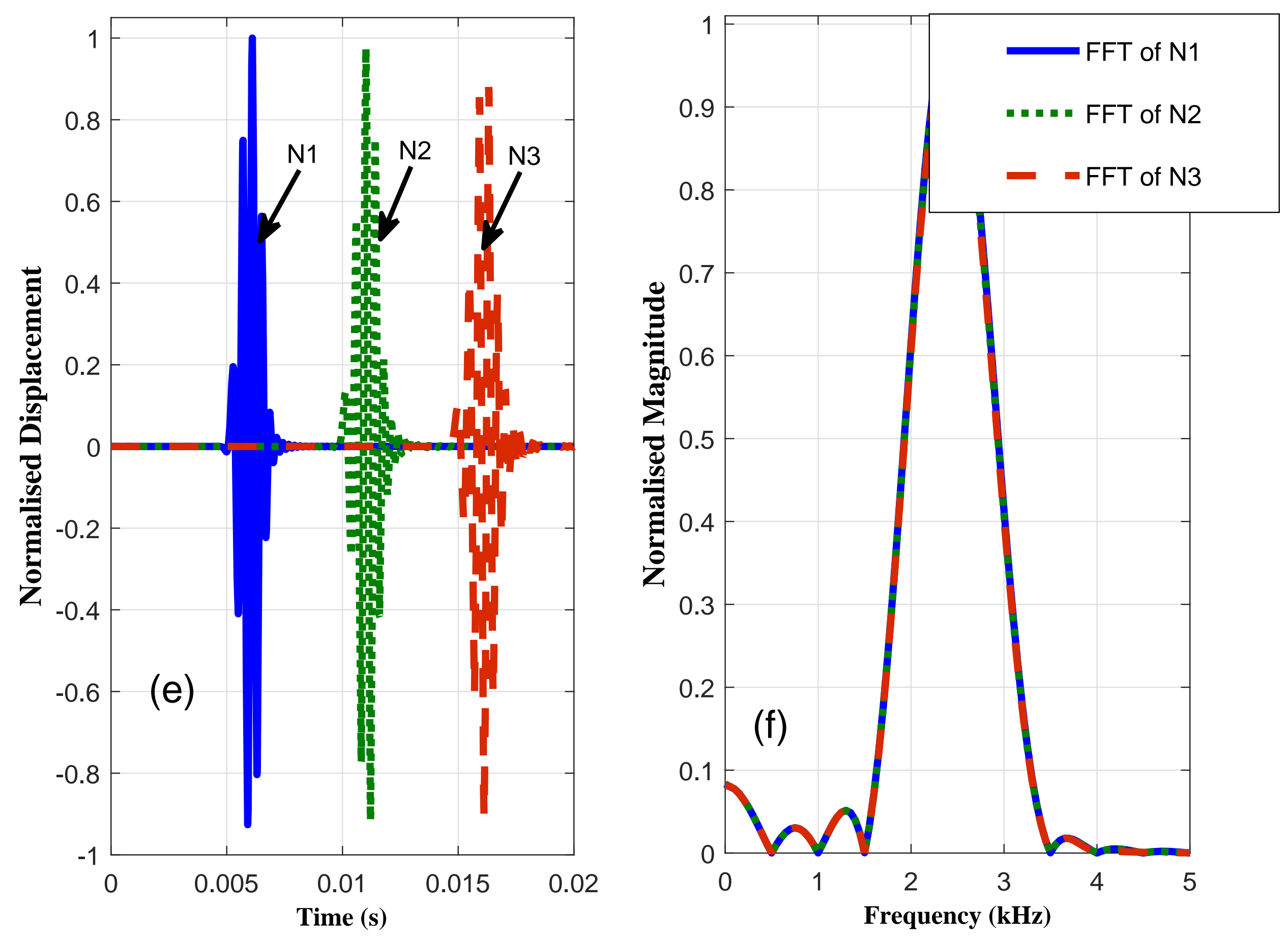

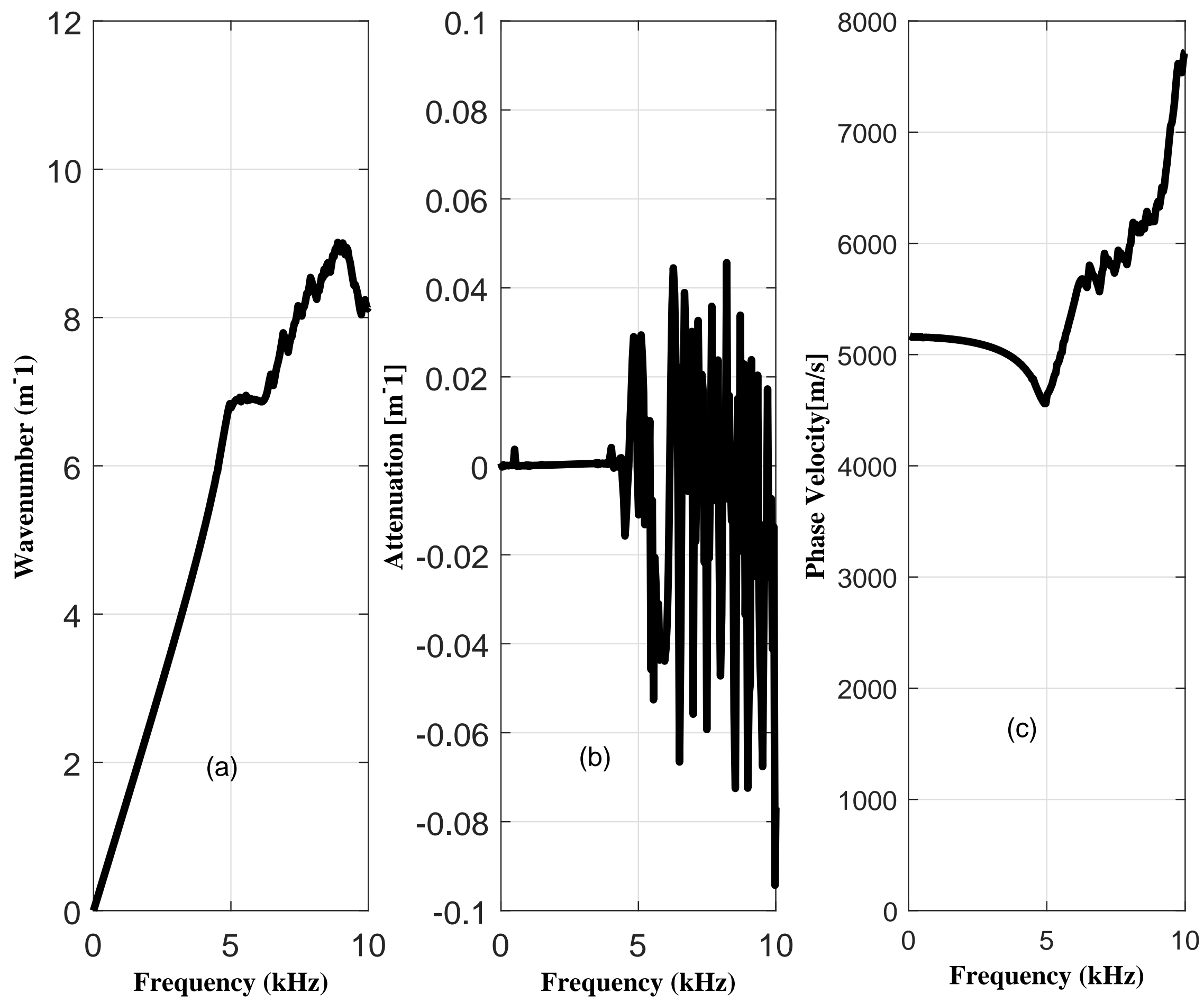


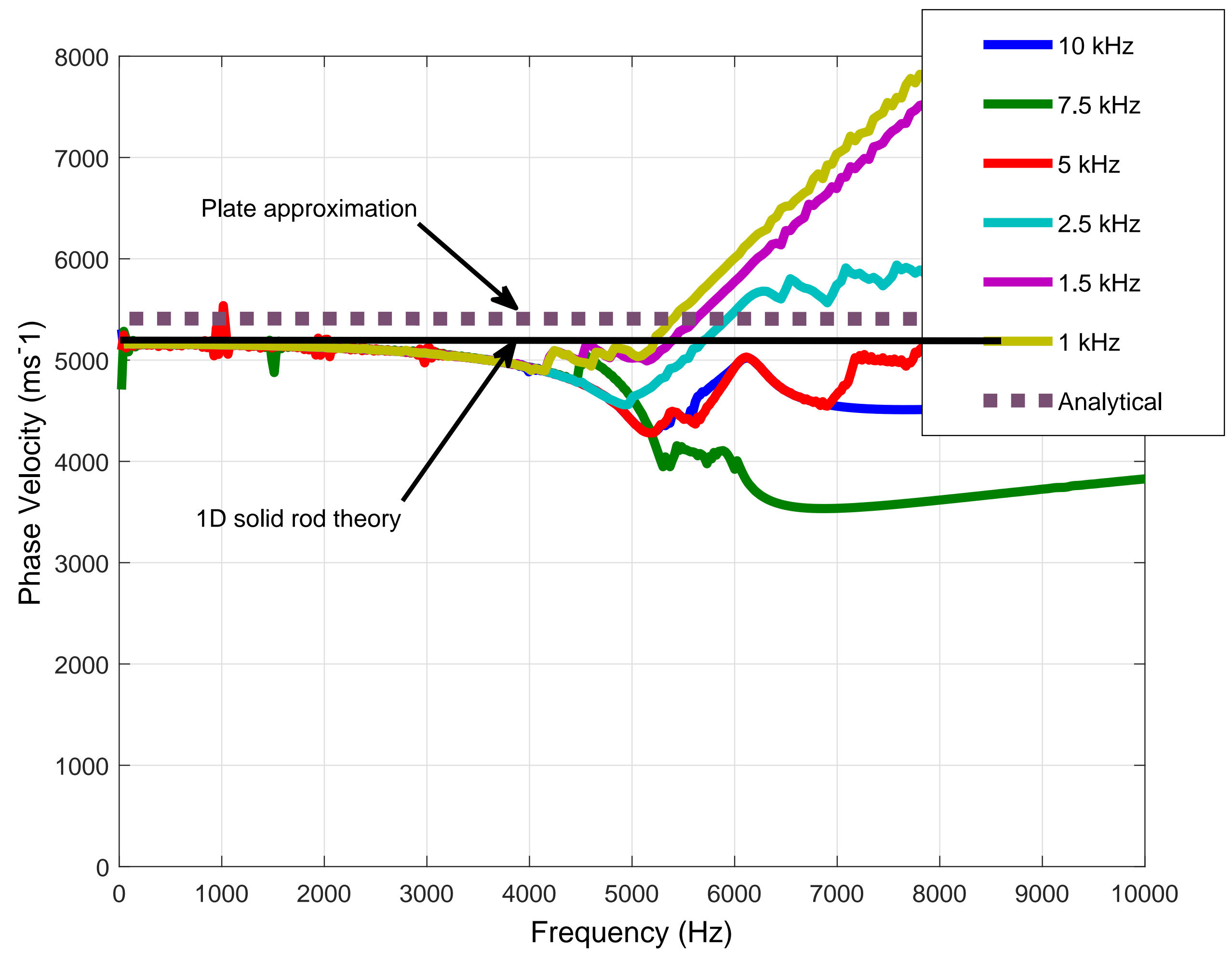




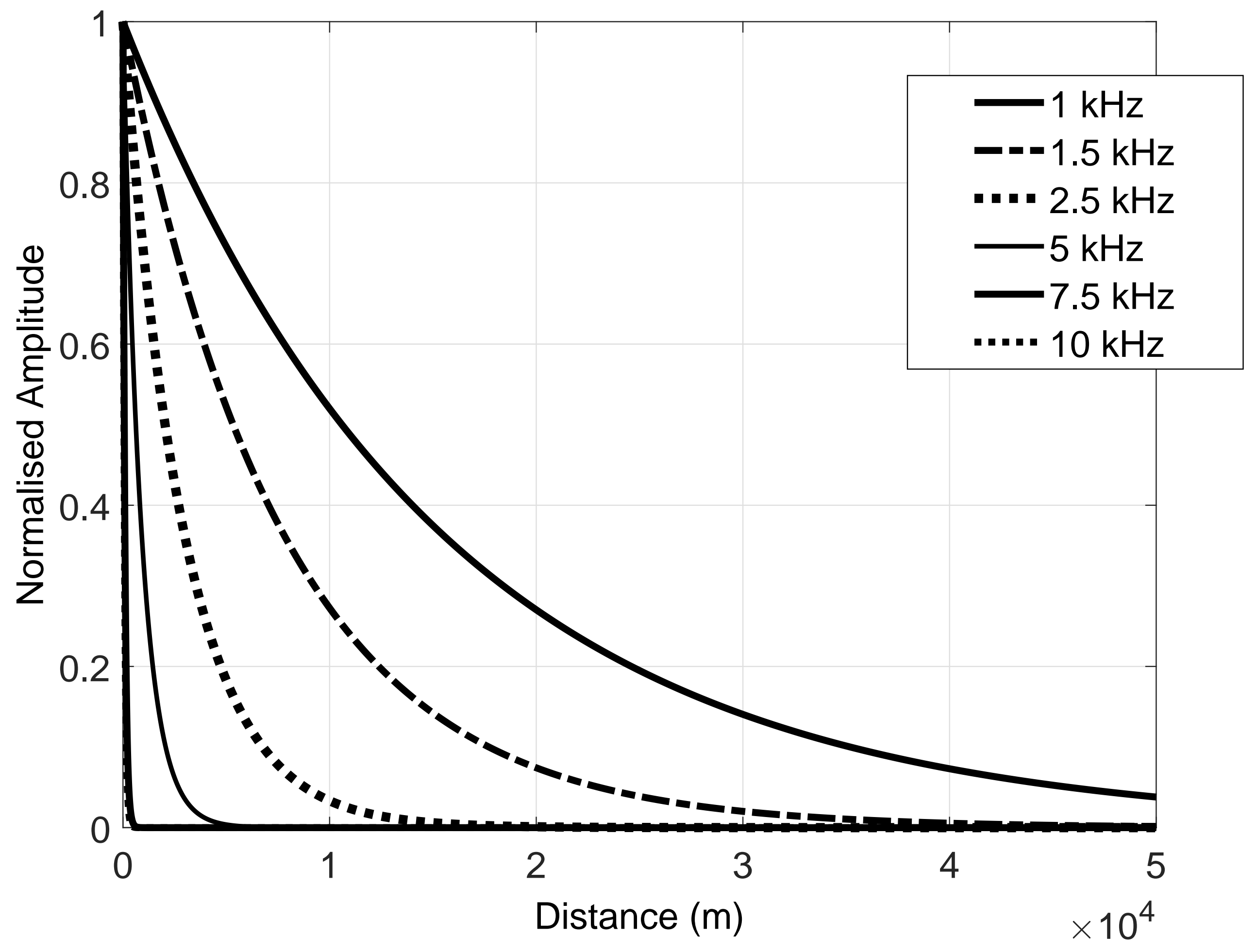



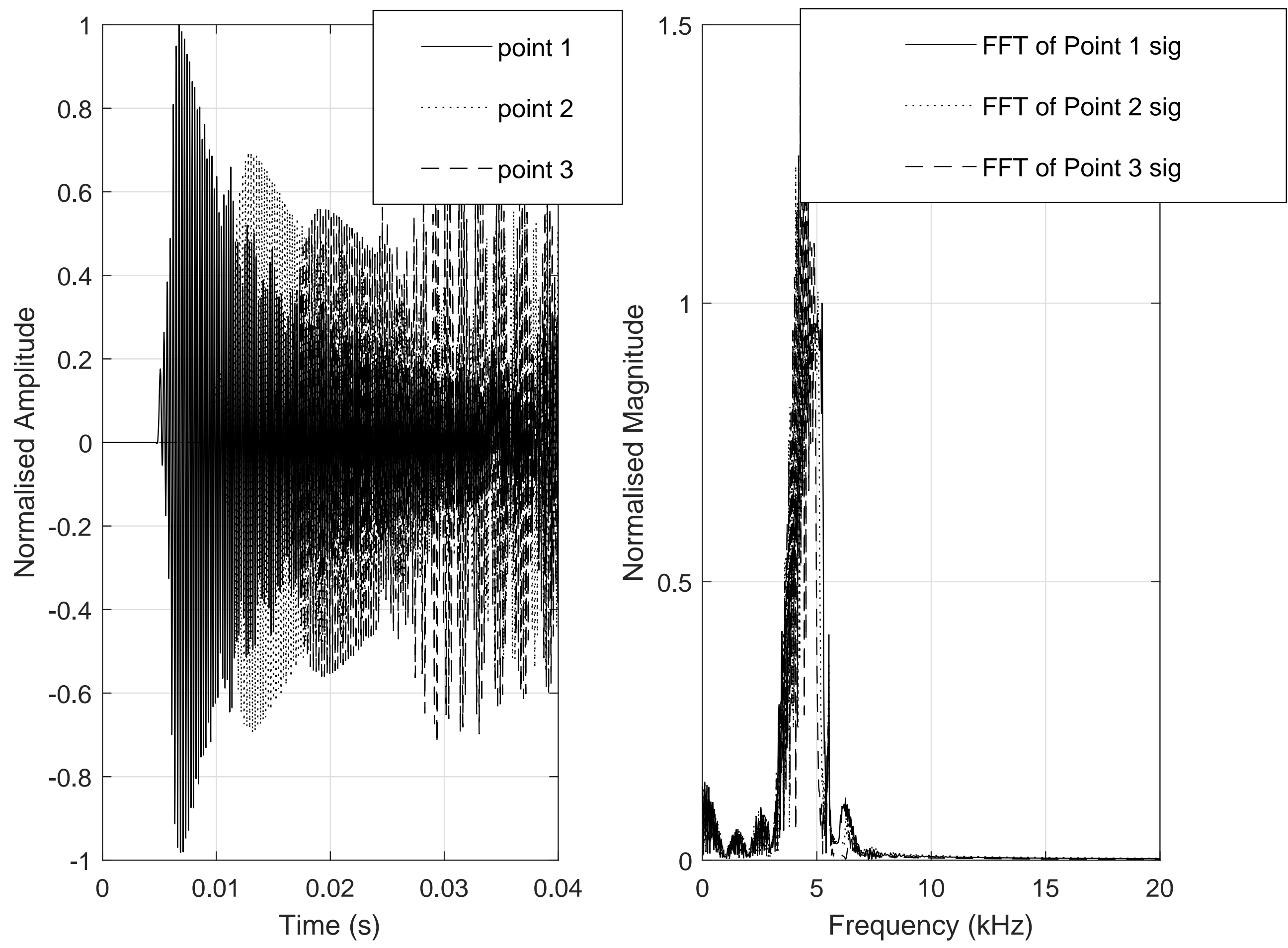

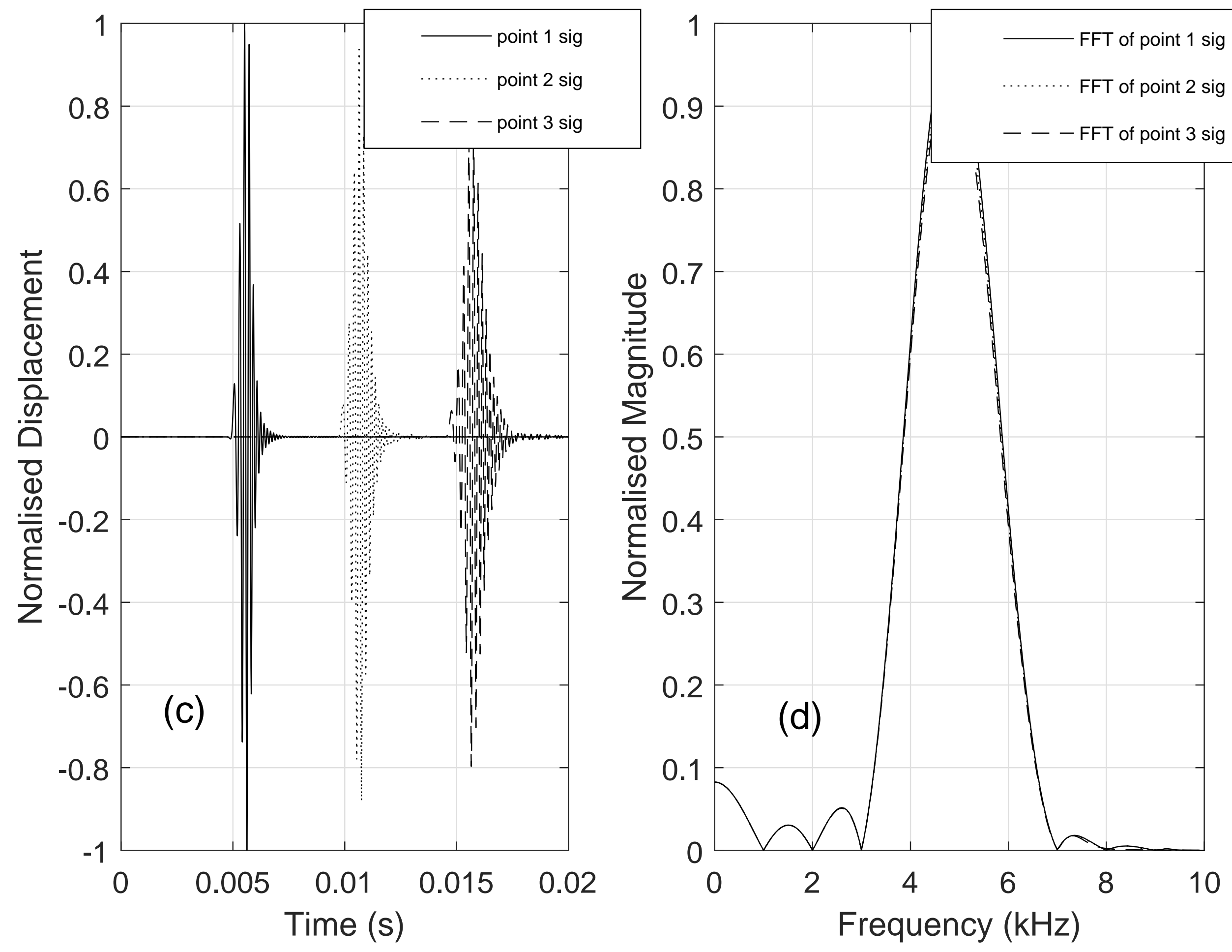

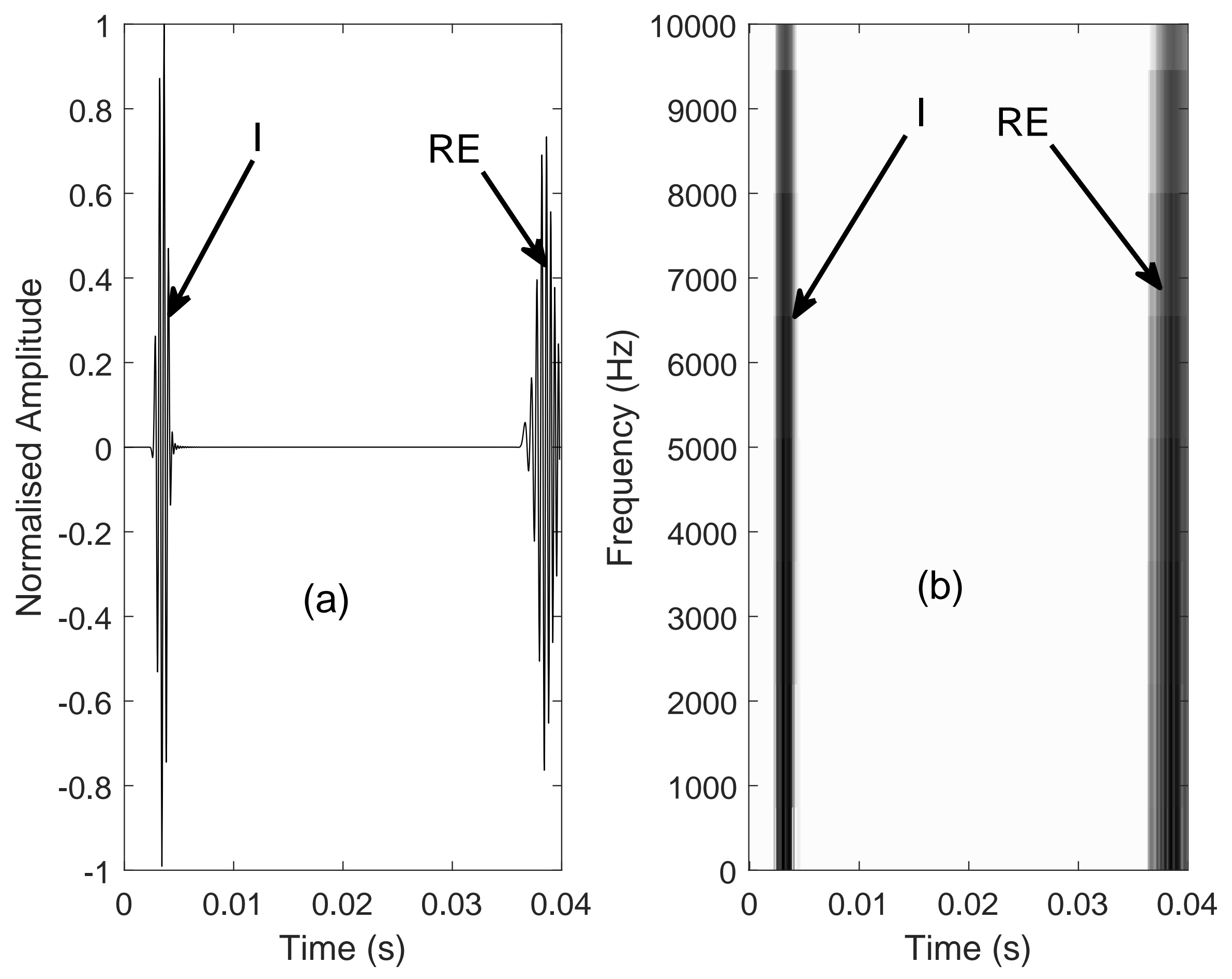

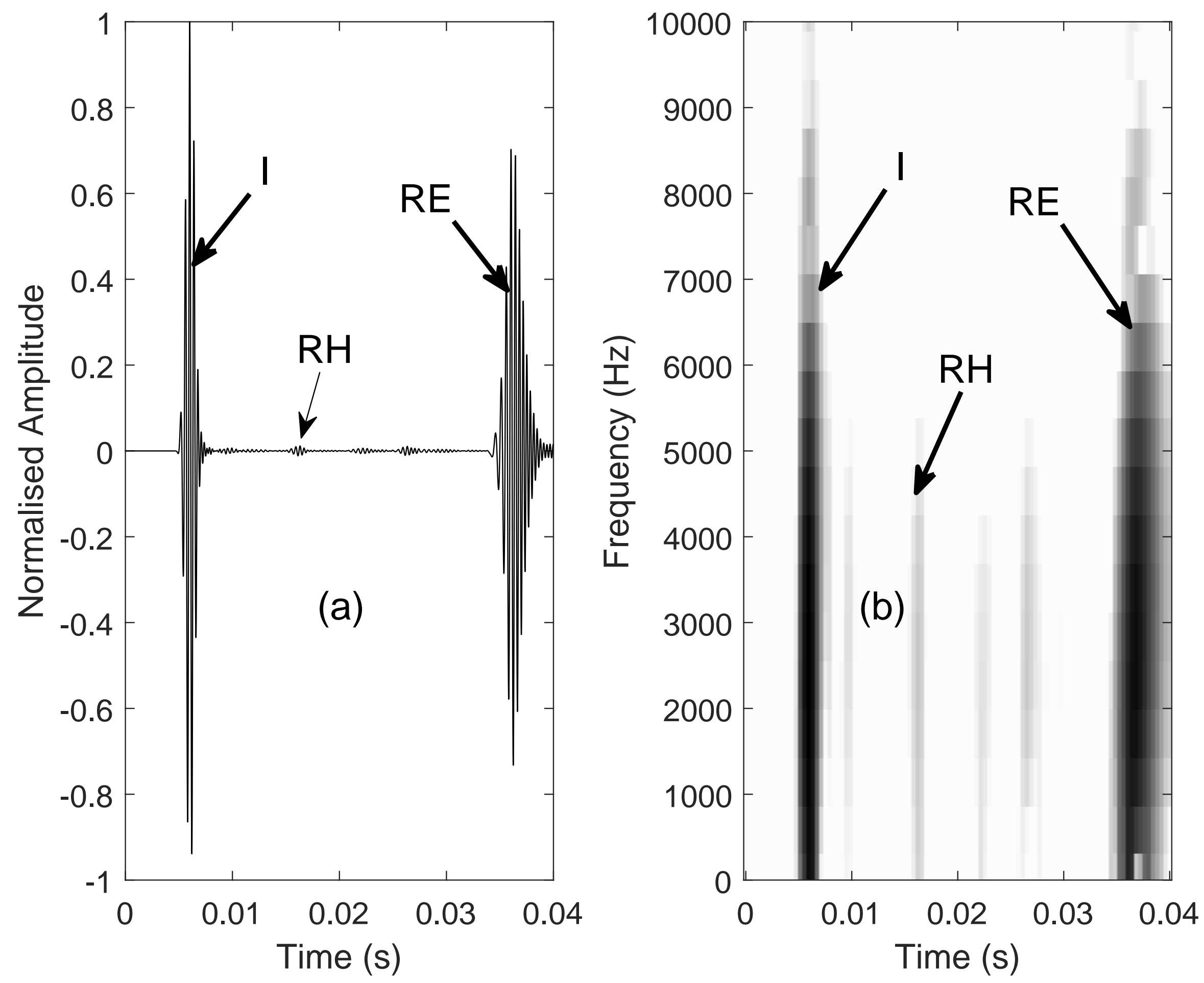

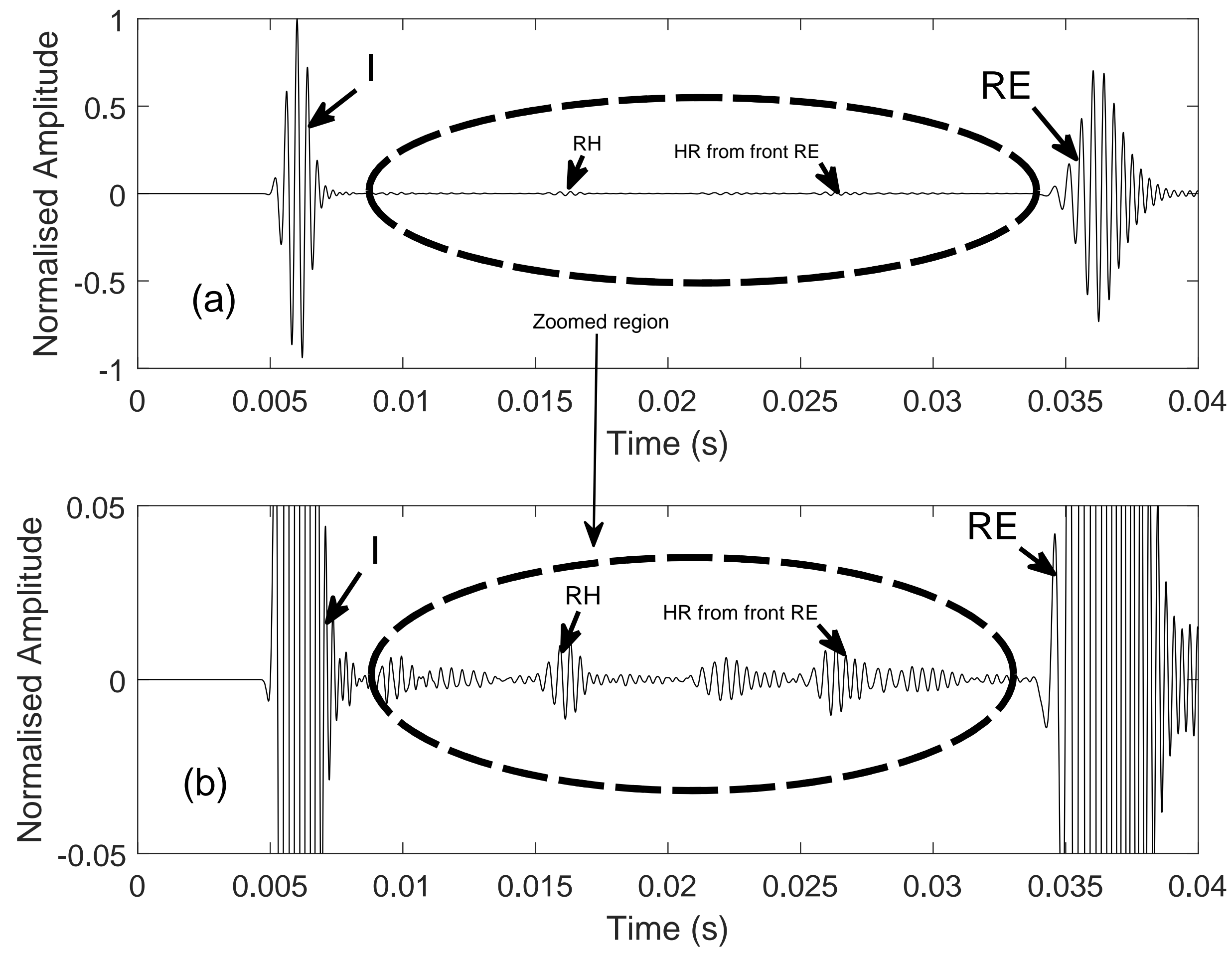

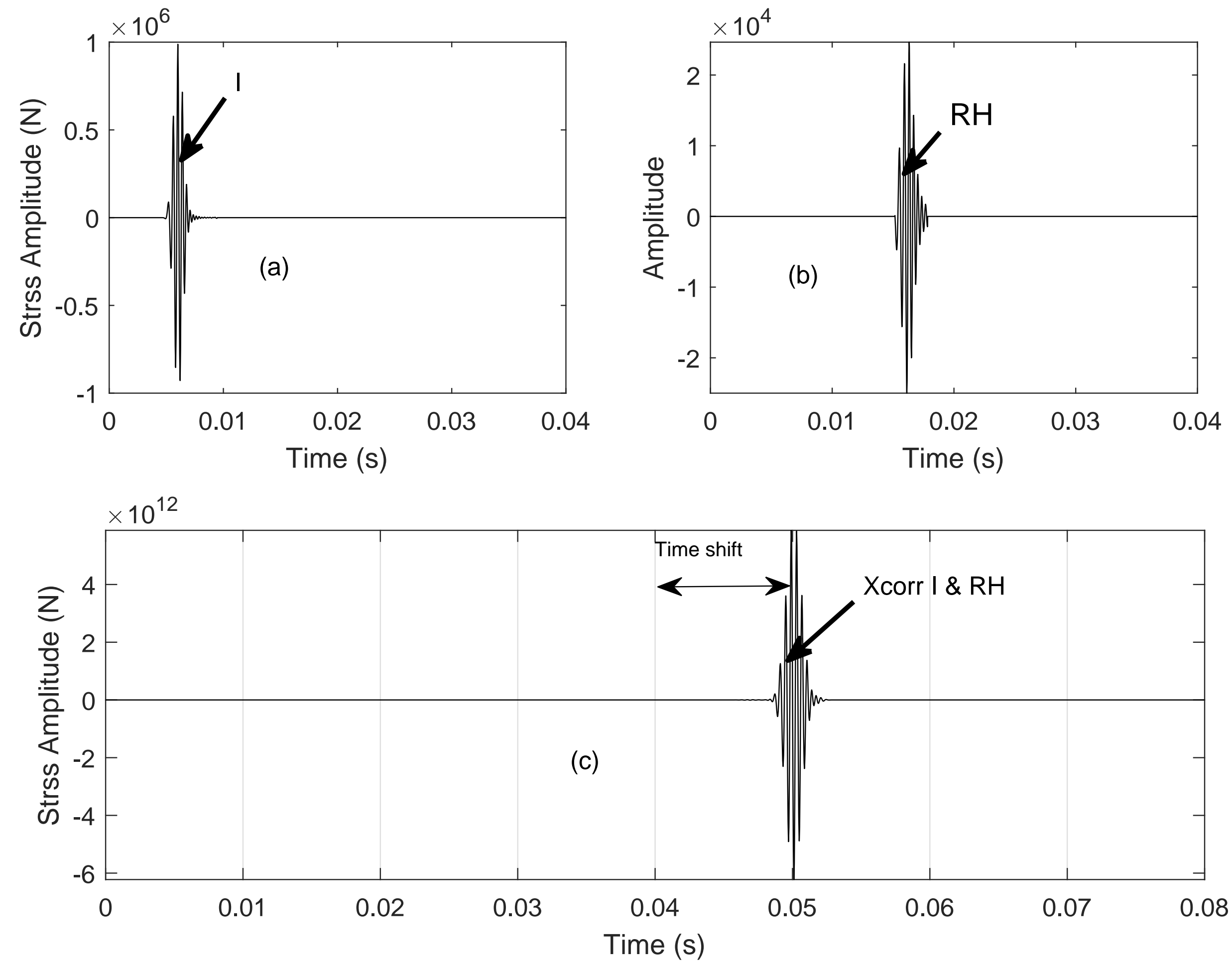

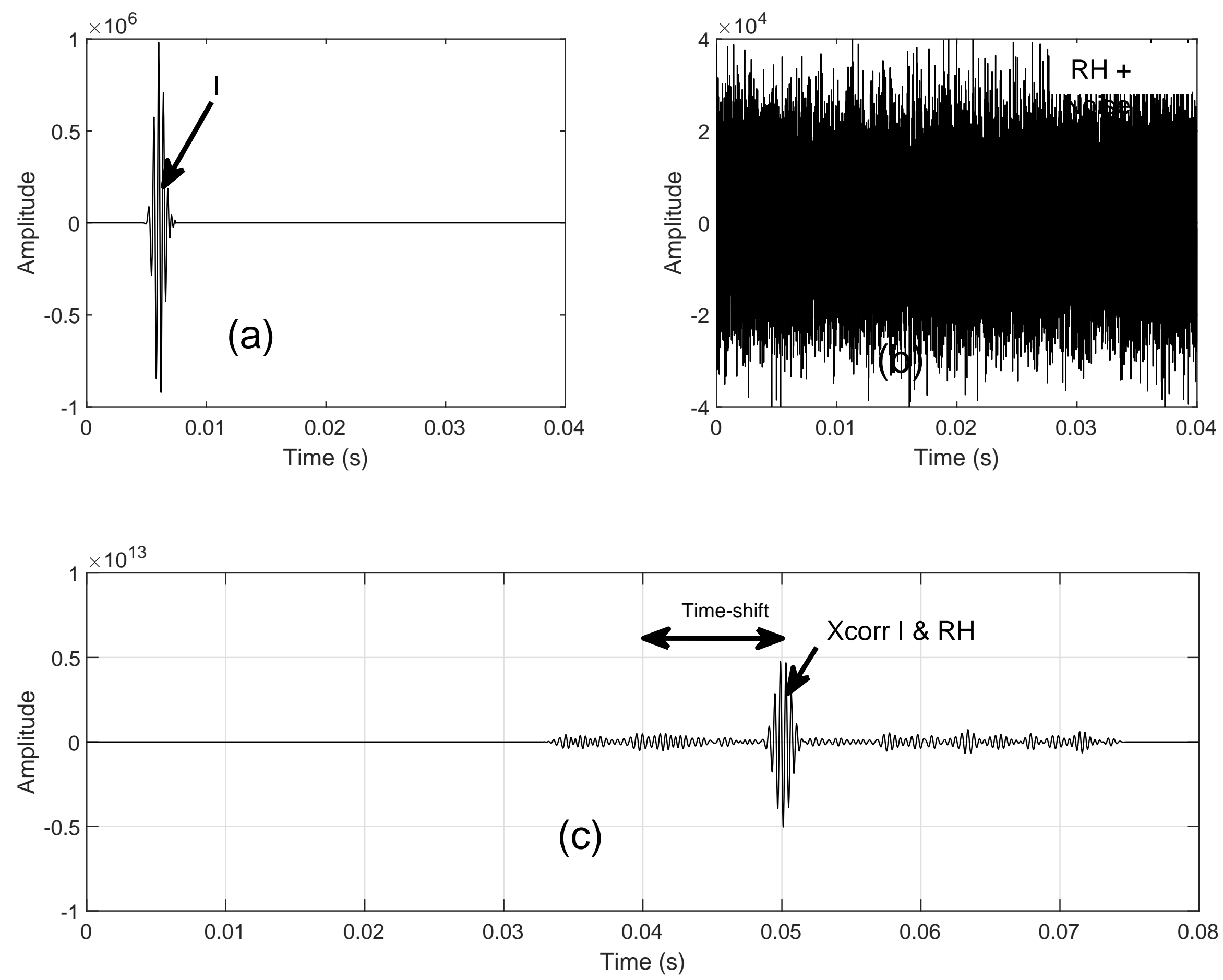


\section{Paper Word figures}

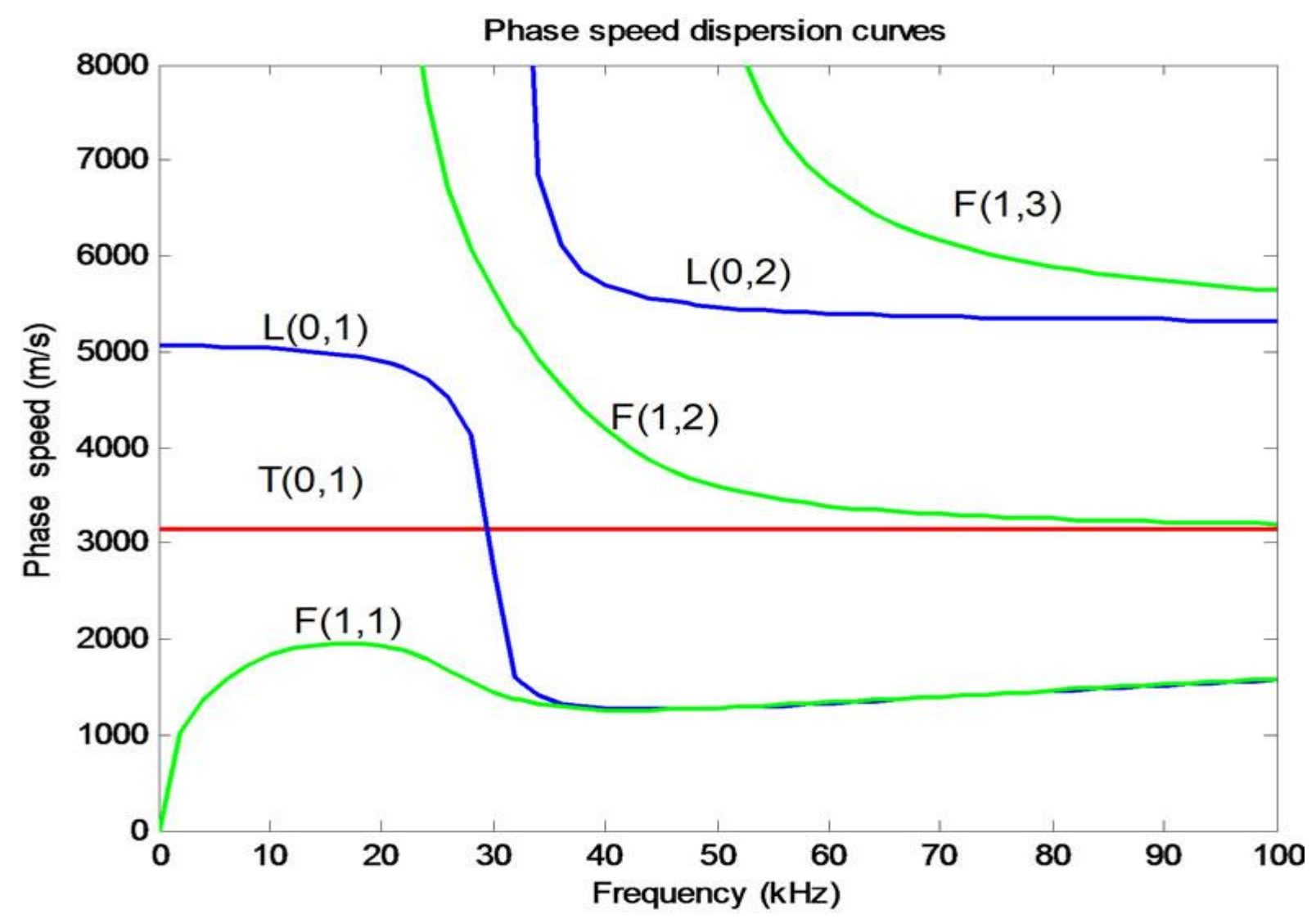

Figure 1

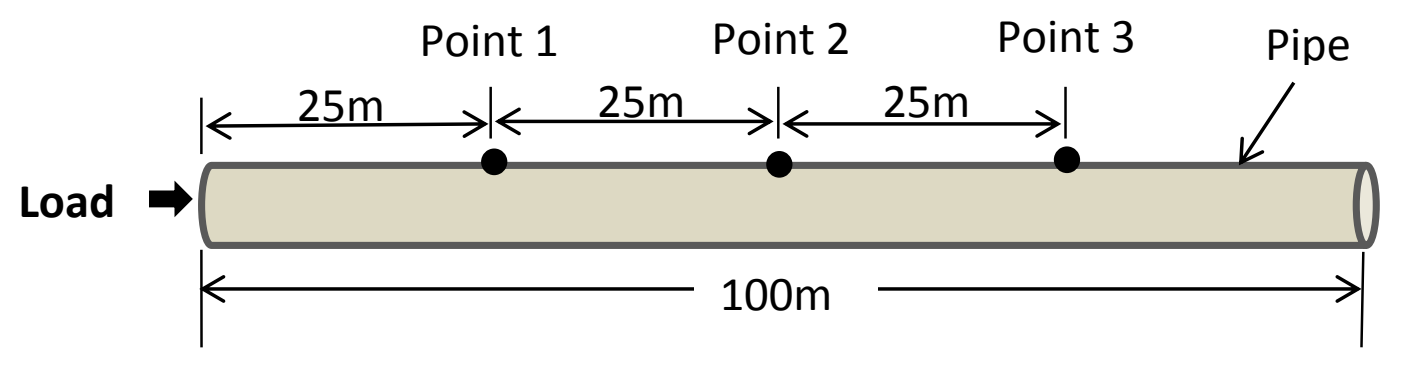

Figure 2 


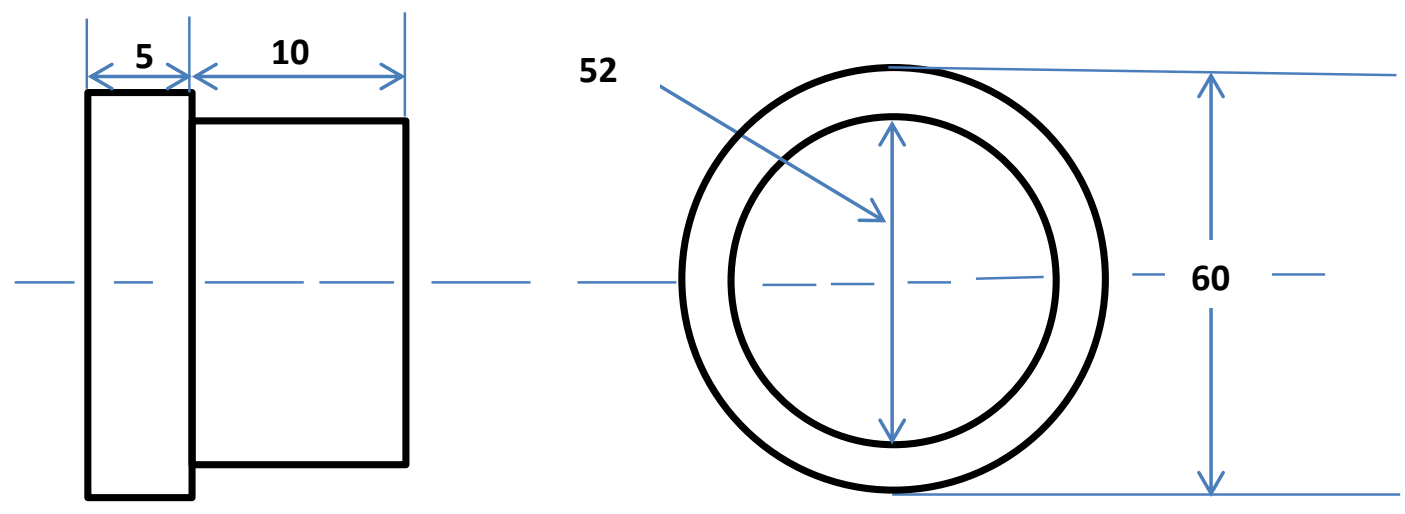

Figure 14

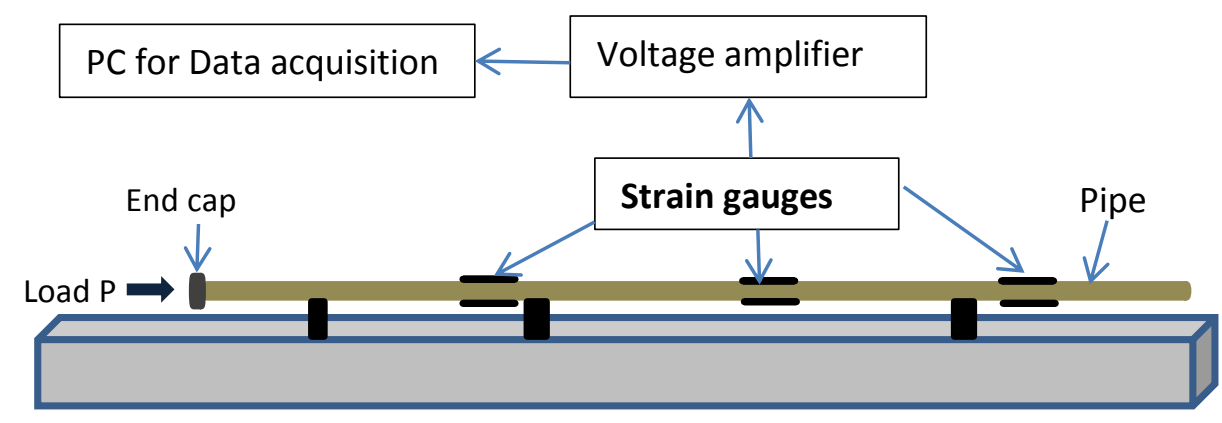

Figure 16 
Paper Tables

Table 1 Some major commercial application of guided wave technology.

\begin{tabular}{lll}
\hline Company Name & Application area & $\begin{array}{l}\text { Reported inspection } \\
\text { distance (m) }\end{array}$ \\
\hline Guided Ultrasonic Ltd & Rail track, piping & $\begin{array}{l}100 \mathrm{~m} \text { for track, not } \\
\text { specified for pipe }\end{array}$ \\
$\begin{array}{l}\text { TechCorr USA Management } \\
\text { LLC }\end{array}$ & $\begin{array}{l}\text { Oil and gas pipeline, } \\
\text { petrochemical, storage, }\end{array}$ \\
$\begin{array}{l}\text { Wavesinsolids LLC (WINS) } \\
\text { Plant Integrity Ltd }\end{array}$ & $\begin{array}{l}\text { Piping, rail track, bridges } \\
\text { Envirocoustic (NDT) }\end{array}$ & 300 \\
& Oil and gas Piping & 180 \\
UltraWave LRT/Olympus & Piping (buried and & 60 \\
MISTRAS Asset property & insulated) & 182 \\
solution & & 35 \\
\hline
\end{tabular}

Table 2 Variation of reflection coefficients with frequency and circular hole diameter

\begin{tabular}{lllll}
\hline & \multicolumn{4}{c}{ Reflection coefficients } \\
\cline { 2 - 5 } Frequency $(\mathbf{H z})$ & 1 inch hole & 2 inch hole & 3 inch hole & 4 inch hole \\
& & & & \\
1000 & 0.0007 & 0.0047 & 0.0123 & 0.0336 \\
$\mathbf{1 5 0 0}$ & 0.0014 & 0.0074 & 0.0178 & 0.0475 \\
$\mathbf{2 5 0 0}$ & 0.0025 & 0.0134 & 0.0273 & 0.0592 \\
\hline
\end{tabular}




\title{
Long range guided waves for detecting holes in pipelines
}

\author{
Salisu El-Hussein ${ }^{1}$, John J Harrigan ${ }^{2}$, Andrew Starkey ${ }^{1}$ \\ ${ }^{1}$ Lloyd's Register Foundation Centre for Safety and Reliability Engineering, School of \\ Engineering, University of Aberdeen, Aberdeen AB24 3UE, United Kingdom \\ ${ }^{2}$ Amec Foster Wheeler, Pavilion 2, City View, Craigshaw Drive, Aberdeen AB12 3BE, \\ United Kingdom
}

\begin{abstract}
The use of low frequency (less than $10 \mathrm{kHz}$ ) guided waves for structural health monitoring (SHM) against third party intrusion into long pipelines is investigated. A guided wave $(\mathrm{GW})$ is sent along a pipeline and its reflection is used to detect the presence of a drilled hole or a branch connected to the pipeline. Finite element (FE) analysis was conducted on $305 \mathrm{~mm}$ (12 inch) outside diameter, $12 \mathrm{~mm}$ wall thickness mild steel pipe. The effects of pipe diameter and thickness on the GW propagation characteristics are illustrated. It is shown that the use of low frequencies have a lower attenuation and therefore are suitable for long range propagation. It is shown that GWs have the potential to detect holes over 100s of meters of pipeline
\end{abstract}

Keywords: Guided waves, structural health monitoring, pipelines

\section{Introduction and background}

The motivation here is the rapid inspection of long pipelines for the detection of large features such as holes or branch connections that are typically $50 \mathrm{~mm}$ to $100 \mathrm{~mm}$ in diameter. Nigeria, for example, has large networks of surface pipelines which are subjected to frequent vandalism and product theft (Ogwu 2011, Udofia 2012). Vandalism results in a damaged pipeline while product theft is initiated through a drilled hole on the pipeline. The challenge is the maximum distance that can be monitored from a single transducer location. To date, GW technology has been applied predominantly in the ultrasonic regime due to the requirement of high resolution to detect small features such as cracks and corrosion patches. 
High resolution comes at the expense of propagation distance (Løvstad 2012). The theory and mathematical formulations of GW technology are well documented, e.g. (Graff 1975, Loveday 2012, Lowe 2001, Rose 2002, Rose 2004b, Rose 2009) and will not be repeated here. GWs are sensitive both to changes in cross-section and material properties (Rose 2004b) and have the potential of providing long distance pipeline inspection with $100 \%$ circumferential monitoring (Davies 2006). Many studies have been carried out on the long distance (up to $100 \mathrm{~m}$ ) inspection of pipes and other tubular structures (Gsell 2004, Li 2006, Long 2003b, Lowe 1998, Shin 1999). Such an approach has the potential of reducing the overall life-cycle cost of a structure and in addition enhances operational safety and reliability (Lee 2013). The major challenges associated with the application of GWs for long range SHM of pipelines are the formation of multiple modes, dispersion and attenuation as detailed in (Alleyne 1992).

The possible GW modes in a circular structure are longitudinal, torsional and flexural, represented as $L(0, m), T(0, m)$ and $F(n, m)$ respectively (Long 2003b). The letter ' $n$ ' represents the circumferential order and ' $m$ ' the harmonic counter of the same mode family. The higher the value of ' $m$ ' the more complicated the mode becomes. The $F(n, m)$ mode shapes vary in sinusoidal form in the circumferential direction with $n=1$ having one circumferential cycle displacement, $n=2$ having two circumferential displacements and so on (Li 2006). For each mode the phase velocity and attenuation are dependent on frequency. The fundamental modes, $L(0,1)$ and $T(0,1)$ have uniform stress distribution across the entire section. They are found to have several advantages over the other modes such as ease of excitation and reception, uniform sensitivity and low dispersion at low frequency (Fletcher 2012). Detailed descriptions of GW modes and frequency considerations are given in e.g. Refs. (Long 2003, Rose 2000). Attenuation and dispersion are major obstacles to long range application of GWs. The major sources of GW attenuation were identified in (Lee 1934) as leakage into the 
surrounding media, scattering at pipe joints and fittings and absorption. The leakage is dependent on the mismatch between the acoustic impedance of the waveguide and the embedding medium (Long 2003a). Scattering induces attenuation as a result of energy loss due to partial reflections which takes place within the waveguide while material damping results in absorption. GWs usually propagate in packets of multiple frequencies. Each frequency component has a different velocity, causing dispersion which in turn leads to a poor signal to noise ratio (Izadpanah 2008).

In the last two decades substantial progress has been made in GW theory and application (Cho 2012). Guided wave SHM has been extensively researched for short $(<1 \mathrm{~m})$, medium (about $5 \mathrm{~m}$ ) and long (about 100m) range applications (Cawley 2002). Frequency is the major difference between the application ranges. Generally, frequencies in the MHz regime are used for short range and above $250 \mathrm{kHz}$ for medium range while below $100 \mathrm{kHz}$ are used for long range. In the short range, GW potential medical applications such as cortical bone assessment and imaging has also been reported (Gsell 2004, Ta 2006, Xu 2010, Yan 2010). GW long range testing has been successfully applied to elongated structures such as aircraft fuselages (Achenbach 1974, Croxford 2007, Raghavan 2007), rail road tracks (Loveday 2012), pipes (Rose 2009) and rock bolts (Beard 2003). The potential of GWs to detect fouling in pipes has also been reported (Hay 2003, Lohr 2003) and used as a means of determining the elastic properties of materials (Walley 2005).

This work investigates the potential of low frequency (below $10 \mathrm{kHz}$ ) GWs for monitoring of third party intrusion of pipelines over distances of the order of kilometres. The interaction of a GW with drilled holes of various sizes was simulated. The objective is to investigate the ability of low frequency GWs to detect a hole that has been drilled in a pipeline for the purpose of siphoning petroleum product. A drilled hole followed by a welded small diameter 
branch pipe with a valve is one of the means used to steal from pipelines. Usually, a $2-4$ in size hose is used for this purpose. The potential for long distance propagation in a pipeline with multiple welds is also investigated. The $L(0, m)$ mode is employed for its advantages in relation to (i) long distance propagation, (ii) ease of excitation and (iii) $100 \%$ circumferential monitoring (Cawley 2002, Mijarez 2013). The FE Simulations are supported by small-scale test results.

\section{Long range guided wave application}

$\mathrm{GW}$ is currently being applied in short $(<5 \mathrm{~m}$ ), medium (up to $5 \mathrm{~m}$ ) and long $(>5 \mathrm{~m}$ ) range depending on the frequency used (Lowe 2006), and commercial software is available to that end. The predominant application areas have been on elongated structures such as pipeline and rail line networks. A GW rail inspection system with the potential to inspect $100 \mathrm{~m}$ of rail from a single position was jointly developed by Imperial College and Guided Ultrasonics Ltd (Wilcox 2003). A $1 \mathrm{~km} \mathrm{GW}$ monitoring range has been reported for the detection of breaks in the railway line (Burger 2012). A portable GW rail inspection system was developed at Pennsylvania State University using electromagnetic acoustics transducer (EMATs) (Rose 2004a). GW has also being used for defect detection in bones (Xu 2010), plate (Davies 2006) and rock bolt testing (Yan 2010) for mine roof support. In the area of pipeline inspections there are many commercial vendors such as Plant integrity Ltd, Wavesinsolids LLC (WINS), MISTRAS Asset Property Solution, etc., with varying reported inspection distance ranging from $35 \mathrm{~m}$ to $300 \mathrm{~m}$. The reported inspection distance on pipelines is still limited to 10 s of meters due to the higher frequencies used (i.e. above 200 $\mathrm{kHz}$ ). Table 1 enumerates some of the major commercial GW vendors and their reported monitoring range. To date GW application in the low frequency region is yet to be fully exploited. As human ingenuity in the area of inspection and monitoring continues to grow, 
understanding the behaviour of GW at low frequency could expand its application boundary especially in elongated structures. FE analysis of GW propagation in pipes can provide this understanding to extend the current inspection distance.

\section{Low frequency guided wave potential propagation distance along a pipeline}

The application of guided wave described above is predominantly at ultrasonic frequency region (i.e. > $200 \mathrm{kHz}$ ) where the wavelength is small and comparable to the pipe thickness. This causes the propagating guided wave to be scattered by minute features (e.g. corrosion patches) along the pipeline to result in signal energy attenuation. At low frequency (e.g. $<10$ $\mathrm{kHz}$ ) the wavelength is large compared to the pipe dimension which limit the interaction (i.e. reflection) of the guided wave with small features such as corrosion induced surface roughness.

\section{Guided wave mode selection}

Pipes are waveguides which can support three guided wave modes (i.e. longitudinal, torsional and flexural) which are distinguished from one another by their distinct mode shapes. A guided wave mode is a unique type of wave with its own unique characteristics of displacement fields across the thickness of a waveguide. In general, modes with uniform stress distribution across the entire section and with simple mode shape are of most interest as they are easier to excite and receive.

For long range application the dispersion and attenuation characteristics over long distances are the most important considerations. Mode excitation in its non-dispersive region will allow signal pulse shape retention while lower attenuation will ensure signal energy retention with propagation distance. In this regard, the fundamental modes, $L(0,1)$ and $T(0,1)$ are found to have several advantages over their respective higher order modes and $F(n, m)$ 
mode family (Fletcher 2010). Both modes exist at all frequencies and exhibit low dispersion at lower frequencies. For example, the $T(0,1)$ mode is completely non-dispersive in the frequency range shown. This can easily be seen from the dispersion curve shown in Fig. 1. The $L(0,1)$ mode at low frequency (e.g. below $20 \mathrm{kHz}$ ) has little dispersion and the next higher order mode (i.e. $L(0,2)$ ) exists only at a frequency beyond $30 \mathrm{kHz}$ as seen in Fig. 1. Using this window of frequencies (i.e. 0 - $30 \mathrm{kHz}$ ) the $L(0,1)$ mode can then be selected for excitation for ease of signal processing. In this study $L(0,1)$ is chosen over $T(0,1)$ due to easier excitation in laboratory and practical applications, however it can be seen that the $T(0,1)$ mode could also be used to accomplish the same goals. The $L(0,1)$ mode has radial (out-plane) and axial (in-plane) displacement distribution; with axial component being predominant at low frequencies. This ensures minimal leakage to the surroundings and allows longer propagation distance with the sufficient energy.

\section{Finite element modelling of guided wave propagation in pipeline}

\subsection{Model definition}

FEA is an established method for GW simulations (Moser 1999). ABAQUS/Explicit (Abaqus, n.d.) is used here. Initial investigations were carried out on a $100 \mathrm{~m}$ long pipe section. The outer diameter of the pipe is $305 \mathrm{~mm}$ (12 inch) and the wall thickness is $12 \mathrm{~mm}$. A fundamental longitudinal $L(0,1) \mathrm{GW}$ was excited in the FE model by applying a uniform pressure pulse load at one end of the pipe, as illustrated in Fig. 2. The applied signals (pressure histories) were tone-bursts modified with a Hanning window according to

$$
s(t)=\left\{\begin{array}{cc}
0.5 \sin (\omega t)\left[1-\cos \left(\frac{\omega t}{N_{c}}\right)\right] & 0 \leq T_{c} \\
0 & t>T_{c}
\end{array}\right.
$$


where $\omega=2 \pi f$ is the centre frequency of the tone-burst in $\operatorname{rad} . \mathrm{s}^{-1}, t$ is the time. $N_{c}$ is the number of toneburst cycles and $T_{c}=2 \pi N_{c} / \omega$ is the total period of $s(t)$. Fig. 3 (a) shows a modified 5 cycle tone-burst with a centre frequency of $2.5 \mathrm{kHz}$. Fig. 3 (b) is the corresponding frequency spectrum from which the Hanning window has helped to suppress the side lobes present when raw tone bursts are used. 3-D solid, linear brick elements with reduced integration (C3D8R) were employed to model the pipe. A mesh size of $12 \mathrm{~mm}$ was used in the longitudinal direction after a mesh convergence analysis. The automatic time step $(\Delta t)$ option was used, which stabilized at $6.7633 \times 10^{-7} \mathrm{~s}$. The material properties defined for the pipe material are the elastic modulus of $209 \mathrm{GPa}$ and density of $7850 \mathrm{~kg} \mathrm{~m}^{-3}$ with a Poisson's ratio of 0.3 . Three points for analysis were defined along the $100 \mathrm{~m}$ long pipe at 25 $\mathrm{m}, 50 \mathrm{~m}$ and $75 \mathrm{~m}$ from the excitation end as shown in Fig. 1.

\subsection{Results and discussion}

Energy balance analysis was first carried out to ensure that there is no loss in the model (i.e. all energies are accounted for). Stress and displacement signals were obtained at the three locations along the top surface of the pipe shown in Fig. 2 only, and at no other locations. Since the excitation is axisymmetric, the stress and displacement signals on the outer surface of the pipe are independent of circumferential location. The wave can be monitored by stress, strain or displacement histories in longitudinal or radial directions. Here, displacement in the longitudinal direction is used. The time domain signals recorded at 1 $\mathrm{kHz}, 1.5 \mathrm{kHz}$ and $2.5 \mathrm{kHz}$ and their corresponding frequency spectrum are shown in Fig. 4 (a $-\mathrm{f})$. There is little change in signal shape between the signals at each point along the pipe at the frequencies of $1,1.5$ and $2.5 \mathrm{kHz}$ indicating low dispersion at these frequencies. The pulses that are recorded at the three locations retain their shape and short periods and approximate to one-dimensional time-shifted versions of the excitation signal. However, the 
signals that are produced by the $5 \mathrm{kHz}$ excitation bear little resemblance to this excitation signal as shown in Fig. $5(\mathrm{a}, \mathrm{b})$. This is because the wavelength (about $1.03 \mathrm{~m}$ ) of the signal at $5 \mathrm{kHz}$ is comparable to the mean circumferential diameter length $(0.96 \mathrm{~m})$ of the model pipe. From Fig. 5 (a), the displacement signal at $5 \mathrm{kHz}$ is dispersive to the extent that the signal did not return to zero after doubling the simulation time (from 0.02 to $0.04 \mathrm{~s}$ ). However, when the pipe outside diameter is reduced by half (from 12 to 6 in), the dispersion is significantly reduced as shown in Fig. 5 (c, d). In addition, the FE model was checked with different discretization parameters (i.e. 10, 12, 24, 40 and $60 \mathrm{~mm}$ ) in order to ensure that this dispersion at $5 \mathrm{kHz}$ was not due to the mesh size. This illustrates that at a frequency which has a wavelength similar to the pipe circumference the signal generated is dispersive. Such frequencies need to be avoided for long range GW applications.

The signal analysis at the frequencies considered indicates similarity with one-dimensional (1-D) wave propagation along a solid bar. This is due to the large wavelength involved at these low frequencies. Hence, the propagation characteristics of the GW in this research are obtained using 1-D wave approximation. For one-dimensional wave propagation in long, slender structures, the propagation coefficient is defined as

$$
\varkappa(\omega)=\alpha(\omega)+\mathrm{ik}(\omega)=\alpha(\omega)+i \frac{\omega}{c(\omega)}
$$

where $\omega$ is angular frequency, $\alpha(\omega)$ is the attenuation coefficient, $k(\omega)$ is the wave number, $c(\omega)$ is the phase velocity and the tilde denotes a complex variable. Assuming that the displacement history of a pulse signal is recorded completely at two locations, giving two records $u_{1}(t)$ and $u_{2}(t)$ e.g. as illustrated by the readings from points 1 and 2 in Fig. 2 . Then, in the frequency domain, 


$$
t_{2}(\omega)=t_{1}(\omega) e^{-\gamma(\omega) x}
$$

where $t_{1}(\omega)$ and $\succsim t_{2}(\omega)$ are the Fourier transforms of $u_{1}(t)$ and $u_{2}(t)$ respectively and $x$ is the distance between the 2 points. The wavenumber, attenuation and phase velocity were calculated for each centre frequency using the FE displacement signals from points 1 and 2 and Eqs. (2) and (3). The plot of wavenumber, attenuation and phase velocity against frequency for $2.5 \mathrm{kHz}$ is shown in Fig. 6 (a-c). These phase velocities are compared with the Raleigh-Lamb frequency equation for the symmetric longitudinal wave (i.e. the "analytical" curve in Fig. 7). The Rayleigh-Lamb equation predicts a phase velocity of $5400 \mathrm{~ms}^{-1}$ as frequency tends to zero. The FE predictions give a phase velocity that tends to $5160 \mathrm{~ms}^{-1}$ as frequency tends to zero, which agrees more closely with the 1-D wave phase velocity for a solid circular rod, which is $5159 \mathrm{~ms}^{-1}$ for a rod of the same material properties. This indicates that at low frequencies, the Rayleigh Lamb equation (derived for plates) is not the most appropriate wave model for cylindrical tubes with a diameter to thickness ratio of 25 . For each centre frequency, the phase velocity remains almost constant at about $5160 \mathrm{~ms}^{-1}$ up to about $4 \mathrm{kHz}$. Beyond this point, the phase velocity becomes unrealistic partly due to the low magnitude of the signals at this point for some frequencies (e.g. 1- $2.5 \mathrm{kHz}$ ) and partly due to the occurrence of resonances at some frequencies.

The FE predicted attenuations were used to calculate the potential monitoring distance at each centre frequency. Attenuation of a wave signal is in general frequency dependent. At high frequencies, higher attenuation and scattering occur when the wavelength is similar in size to the structural features. As frequency reduces, attenuation is correspondingly reduced. Frequency dependent attenuation also results from energy loss during wave motion as material particles vibrate back and forth. The non-frequency dependent attenuation results 
from friction and thermal conductivity. Fig. 8 has been produced to illustrate the attenuation of sinusoidal waveforms with different frequencies that is predicted using the attenuation coefficient calculated from the FE simulations. The normalized amplitude is simply calculated using

$$
A(x)=A_{0} e^{-\alpha x}
$$

where $A_{0}$ is the amplitude at $x=0$ and $A(x)$ is the amplitude after the wave has travelled a distance $x$. The attenuation that is present in the FE model is artificially introduced by the FE package used (i.e. ABAQUS/Explicit) for the stability of the model. In ABAQUS/Explicit analysis, a small amount of numerical damping is introduced by default in the form of quadratic and linear bulk viscosity to control the high frequency oscillations. Linear bulk viscosity is introduced to damp the 'ringing' in the highest element frequency known as truncation frequency damping. Quadratic bulk viscosity is introduced to prevent elements from collapsing under extremely high velocity gradients (Abaqus n.d.). However this damping therefore will affect the overall attenuation as without it no energy in the propagating signal would be lost. The results thus obtained will be compared against experimental results presented later in the paper. From Fig. 8 it can be seen that in theory the longitudinal waves can propagate long distances and still retain a substantial percentage of their original energy.

\section{Effect of drilled holes on guided waves}

FE models were created in order to examine the effect of holes in the pipeline ranging from $1-4$ in diameter. When the wave signal reaches the hole, some part of it is reflected back while the majority of the wave is transmitted beyond the hole. An initial investigation 
revealed that it is easier to detect the reflected wave than to examine the transmitted wave for evidence of the hole. The pipe section considered is similar to that discussed earlier and illustrated in Fig. 2. The pipe length is $100 \mathrm{~m}$, the diameter is 12 in (305 mm) and the thickness is $12 \mathrm{~mm}$. Displacement histories are recorded at Point 1 of Fig. 2 and the hole is positioned at Point 2. First, the simulation was carried out with a free pipe, without a hole. For comparison, the displacement history and its spectrograph are plotted for Point 1 in Fig. 9. Within the time window of Fig. 9 (a), the incident wave $(I)$ and its reflection from the far end of the pipe $(R E)$ are seen clearly. Note that the pipe was modeled as having free ends. The spectrograph also shows the incident and end-reflection clearly. No reflections are present except that from the free end.

A drilled circular hole was then created at the mid-length of the $100 \mathrm{~m}$ model pipe. The displacement-time and the spectrograph for Point 1 are plotted in Fig. $10(a, b)$. The incident signal, end reflection and the reflection from the hole are indicated by $I, R E$ and $R H$ respectively. From this Fig. $10(a, b)$, it is difficult to note any reflection. However, when the reflected signal was zoomed and plotted, the reflection from the hole is visible as shown in Fig. 11 (b). The intensity of the spectrum was observed to be increasing with increasing hole diameter. Table 2 gives the summary of reflection coefficients for different excitation frequencies and hole diameters. The reflection coefficient was calculated as the ratio of the amplitude of the incident signal to the reflected signal. Cross correlation signal processing was then used to detect and locate the position of the hole. The cross correlation of $I$ (Fig. 12 (a)) and $R H$ (Fig. 12 (b)) produces a higher peak time shifted signal (Fig. 12 (c)), indicating similarity between them. The figure shows a shift of the peaks in the two impulses, shown at 0.05 in Fig. 12 (c). This indicates a positive time shift of $0.01 \mathrm{~s}$ since the cross correlation for signals occurring at the same time would show peaks at 0.04 (the length of the original impulse windows). 
It should be noted that the reflected signal is small compared to the incident signal and will decay further with the distance from the reflection point. At a certain point this reflected signal may decay below the ambient or operational noise due to routine vibration. However, these observations will not remove the possibility of detecting such small reflection due to the similarity in frequency content between input and reflected signals. Since the input signal pulse is excited with a known frequency the reflected signal can be detected even in the presence of background noise. This is similar to the method used in Radar to detect a flying object in the atmosphere despite the presence of sources of noise in the atmosphere. As an example, the signal in Fig. 12 (b) (i.e. hole reflected signal) was superimposed by a generated random noise and shown in Fig. 13 (b). The input signal is cross-correlated with the combined hole reflection and random noise signals (Fig. 13 (b)). The result of crosscorrelation in Fig. 13 (c) shows that the $R H$ can be detected even in the presence of this background noise. The location of the hole was calculated using the wave velocity at $2.5 \mathrm{kHz}$ centre frequency $\left(5100 \mathrm{~ms}^{-1}\right.$ in Fig. 7) and the time shift of $0.01 \mathrm{~s}$ of the cross-correlated signals which gives the location as $25.5 \mathrm{~m}$. This gives an error of $0.5 \mathrm{~m}$ which is due to the difficulty in getting the exact time shift of the correlated signals.

\section{Experimental validation of FE results}

\subsection{Experiment setup}

A small scale laboratory (lab) test was conducted to validate the FE results. The test was conducted on a 2 in $(50.8 \mathrm{~mm})$ outside diameter schedule 40 mild steel pipe with a wall thickness of $3.2 \mathrm{~mm}$ and a length of $6 \mathrm{~m}$. An end cap shown in Fig. 14 was designed to fit one end of the pipe. Brüel \& kjær impulse hammer type 8206-003 was used for the lab tests. The impulse hammer has a voltage sensitivity of $1.14 \mathrm{mV} / \mathrm{N}$. Fig. 15 (a, b) shows a time history of a typical experimental impulse hammer pulse and its corresponding frequency 
spectrum. From Fig. 15 (b) the signal energy is concentrated below $5 \mathrm{kHz}$.

The impulse hammer was used to apply a longitudinal impact along the pipe through the end cap. The end cap in turn distributes the impact load uniformly around the pipe circumference to generate a longitudinal wave in the pipe. Strain readings were taken at 3 locations $1.5 \mathrm{~m}$ equidistant from one to the next. Fig. 16 shows the layout of the experiment set up. The pipe was supported at 3 positions on a v-shaped support padded with a smoothed and greased plastic to allow free longitudinal movement and prevent signal leakage to the metallic support. At each of the 3 locations 2 foil strain gauges were cemented diametrically opposite each other (180 degrees apart) to minimize the effect of bending. Each strain gauge had a gauge factor of $2.1 \pm 1 \%$, a resistance of $120 \pm 0.3 \Omega$ and a length of $3 \mathrm{~mm}$. The longitudinal strain of the gauge was measured with the aid of a Wheatstone bridge circuit. The active arms of the Wheatstone bridge circuit are made up of the two strain gauges on the pipe while 120 $\Omega$ dummy resistors were used to complete the circuit. The output from each Wheatstone bridge circuit was fed to a voltage amplifier (Fylde Transducer Amplifier type FE-379-TA). The output from the amplifier was then fed to the high speed digital acquisition system module PCI-5105 from National Instruments.

\subsection{Results and discussion}

Fig. 17 shows the time domain comparison of the strain histories from a Lab test and from an FE model of the Lab test. The lines represent the Lab test data and the FE model from the same positions on the pipe. There is a good agreement between the FE predictions and the Lab test data for both the strain histories. The wave propagation characteristics were calculated from the FE and Lab test signals (Fig. 18) using the method for non-overlapping wave. Fig. 18 (a) shows good agreement between FE and Lab test wavenumbers. From fig. 18 (b), the attenuation coefficient of the lab test signal was around zero but contains large 
fluctuations. The FE attenuation remains fairly constant around zero for frequencies below 5 $\mathrm{kHz}$ and rises afterward. The phase velocities from FE and lab test shown in Fig. 18 (c) agree well at about $5150 \mathrm{~ms}^{-1}$. The summary of the results indicated that the FE predicts the test data well. This agreement of the Lab test and FE of the Lab test model validates the earlier FE results on the potential propagation distance of low frequency GW discussed in section 2.

\section{Conclusion}

The results of the simulation show that longitudinal GW at low frequency (below $5 \mathrm{kHz}$ ) has the potential for long distance propagation in pipe with little energy loss. It was shown numerically that GW at this frequency region can propagate up to about $8 \mathrm{~km}$ in a free pipe retaining more than 10 per cent of its initial energy. However, the result presented contains artificial FE damping which is not real and does not include damping in steel which is real. In addition, the presence of weld geometry and other features (e.g. pipe support and surrounding medium) could reduce the reported potential distance that can be achieved in practice and the effects of these will be the focus of further studies. Additionally, simulations indicate that longitudinal guided waves at low frequencies are able to detect circular holes (e.g. 1, 2 and 3 inch) along 12 in. pipelines. The reflections received from drilled hole have the same frequency content as the input signals. This allows the smaller reflected signals to be easily detected and the hole location to be determined using cross correlation. A small scale Laboratory experiment was conducted to validate the FE results. The FE simulations agree well with experimental results. However, in practice attenuation may also be affected by a number of issues such as contact with the ground, pipe supports, protective coatings, surface corrosion, and viscous losses into the internal product. These areas will form the basis of future work in this area, including a full scale trial on pipes with holes introduced, as well as the impact of other issues such as material inside the pipe (including multi-phase flow 
material and liquid mixes of water and petroleum products), and the effect of supports and other surrounding material around the pipe.

\section{Acknowledgement}

The first author wishes to acknowledge the financial support of Petroleum Technology Development Fund (PTDF) - Nigeria.

\section{References}

Abaqus analysis user's manual (n.d.), version 6.12.

Achenbach JD (1974). Wave propagation in elastic solids. Amsterdam: North Holland Publishing Company.

Alleyne DN, Cawley P (1992). Optimization of lamb wave inspection techniques. NDT \& E International; 25:11-22.

Beard MD, Lowe MJS (2003). Non-destructive testing of rock bolts using guided ultrasonic waves. Int. J. Rock Mech. Min. Sci.; 40:527-36.

Burger F A (2012) A practical continuous operating rail breaks detection system using guided waves In: 18th World Conf. on NDT 16-20 April 2012 Durban, South Africa.

Cawley P (2002). Practical long range guided wave inspection - application to pipe and rail. NDE2002, predict.assure.improve, National Seminar of ISNT Chennai, 5 - 7 December 2002.

Cho Y. (2012) Model-Based Guided wave NDE: The Evolution of Guided wave NDE from "Magic" to "Physically Based Engineering Tool”. J. Nondestruct. Eval. 2012; 31:324338, doi 10.1007/s10921-012-051-y.

Croxford, AJ, Wilcox PD, Drinkwater BW, Konstantinidis G. (2007) Strategies for guidedwave structural health monitoring. Proc. R. Soc. Am 2007; 463:2961-81.

Davies J, Simonetti F, Lowe M and Cawley P (2006) Review of synthetically focused guided wave imaging techniques with application to defect sizing AIP Conf. Proc. 820 142(2006) doi:10.1063/1.2184522

Fletcher, S. Lowe, M, Ratassepp, M. and Brett, C, (2010). Detection of axial cracks using focused guided waves, pp. 223-230.

Fletcher S, Lowe M, Ratassepp M, Brett C. (2012) Detection of axial cracks using focused guided waves. J Nondestruct Eval 2012; 31:56-64. DOI 10.1007/s10921-011-0120-x 
Graff KF (1975). Wave motion in elastic solids. Oxford: Clarendon Press; 1975.

Gsell D, Dual J. (2004) Non-destructive evaluation of elastic material properties in anisotropic circular cylindrical structures. Ultrasonics 2004; 43:123-132.

Hay TR, Rose JL (2003). Fouling detection in the food industry using ultrasonic guided waves. Food Control 2003; 14:481-88.

Izadpanah S, Rasheed GR, Sodagar S (2008). Using ultrasonic Guided waves in evaluation of pipes. The 2nd International Conference on Technical Inspection and NDT 2008; (TINDT2008) - Tehran, Iran.

Lee LH, Rajkumar R, Lo LH, Wan, CH, Isa D (2013). Oil and gas pipeline failure prediction system using long range ultrasonic transducers and Euclidean-Support Vector Machines classification approach. Expert Systems with Applications 2013; 40:19251934.

Li J, Rose JL (2006). Natural Beam Focussing of non-axisymmetric guided waves in largediameter pipes. Ultrasonics 2006; 44:35-45.

Liu Z, Liu S, Wu B, Zhang Y, He C (2008). Propagation characteristics of high order longitudinal modes in steel strands and their applications. ActaMechanicaSolidaSinica 2008; 21:573-79.

Lohr KR, Rose JL (2003). Ultrasonic guided wave and acoustic impact methods for pipe fouling detection. J. Food Eng. 2003; 56:315-24.

Long R, Cawley P, Lowe M (2003). Acoustic waves propagation in buried water pipes. Proc. R. Soc. London 2003; 459:2749-70.

Long R, Lowe M, Cawley P (2003). Attenuation characteristics of fundamental modes that propagate in buried iron water pipes. Ultrasonics 2003; 41:509- 519.

Loveday PW (2012). Guided wave inspection and monitoring of railway track. Journal of Non-destructive Evaluation 2012; 31: 303-309.

Løvstad A, Cawley P (2012). The reflection of the fundamental torsional mode from pit clusters in pipes. NDT \& E International 2012; 46: 83-93.

Lowe MJS, Alleyne P, Cawley P (1998). Defect Detection in Pipes using Guided waves. Ultrasonics $1998 ; 36: 147-154$.

Lowe MJS (2001). Guided waves in Structures. In: Braun, Ewins, Rao, San Diego, editors. Wave propagation, Academic Press; 2001, p. 1551-1559. 
Lowe M J S and Cawley P (2006) Guided waves long inspection usage - current commercial capabilities and research directions Department of Mechanical Engineering Imperial College London http://www3.imperial.ac.uk/pls/portallive/docs/1/55745699

Mijarez R, Gaydecki P (2013). Automatic guided wave PPM communication system for potential SHM of flooding members in sub-sea oilrigs. Smart. Mater. Struct. 2013; 22:1-9.

Moser F, Jacobs LJ, Qu J (1999). Modelling elastic wave propagation in waveguide with finite element method. NDT and E Int. 1999; 32:225 - 34.

Ogwu FA (2011). Challenges of oil and gas pipeline network and the role of physical planners in Nigeria. Newcastle University Forum Journal 2011; 10: 41-51.

Raghavan, A, Cesnik, C.E.S (2007). Review of guided wave structural health monitoring. The Shock and Vibration Digest 2007; 29: 91-114.

Rose JL (2000). Guided waves Nuances for Ultrasonic Non-destructive Evaluation. IEEE Trans. Ultrason., Ferroelectr. Freq. Control 2000; 47:575-83.

Rose JL (2002). A Baseline and vision of ultrasonic guided wave inspection potential. Journal of Pressure Vessel Technology 2002; 124:273-282.

Rose JL, Avioli M (2004) Guided wave inspection potential of defects in rail NDT and E Int. 37 174-80

Rose JL (2004). Ultrasonic waves in solid media. New York: Cambridge University Press

Rose JL, Cho Y, Avioli MJ (2009). Next generation guided wave health monitoring for long range inspection of pipes. Journal of Loss Prevention in the Process Industries 2009; 22: 1010-1015.

Shin HJ, Rose JL (1999). Guided wave by axisymmetric and non-axisymmetric surface loading on hollow cylinders. Ultrasonics 1999; 37:355-363.

Ta D, Huang K, Wang W, Wang Y, Le LH (2006). Identification and analysis of multimode guided wave in tibia cortical borne. Ultrasonics 2006; 44:274-84.

Udofia OO, Joel OF (2012). Pipeline vandalism in Nigeria: Recommended best of practice of checking the menace. Society of Petroleum Engineers, SPE Nigerian Annual International Conference and Exhibition Abuja-Nigeria 6-8 August 2012.

Walley SM, Field JE (2005). Elastic propagation in materials. Encyclopaedia of materials Science and Technology 2005; p. 1-7. ISBN: 0-08-043152-6.

Wilcox P, Pavlakovic B, Evans M, Vine K, Cawley P, Lowe M and Alleyne DN (2003) Long range inspection of rail using guided wave AIP Conf. Proc. 657 236-43 
Xu K, Ta D, Wang W (2010). Multiridge-based analysis for separating individual modes from multimodal guided wave signals in long bones. IEEE Trans. Ultrason. Ferroelectr. Freq. Control 2010; 57:2480-90.

Yan F, Royer JR RL, Rose JL (2010). Ultrasonic guided wave imaging techniques in structural health monitoring. J. Intell. Mate. Syst. Struct. 2010; 21:377-84.

Yan F, Royer J R and Rose J L (2010) Ultrasonic guided wave imaging techniques in structural health monitoring J. Intell. Mater. Syst. Struct. 21377 - 84.

Wang, Y. M. Shen, C. J. Zhu, L. X. Sun F. R., (2012). Guided Waves Mode Discrimination in Pipes NDT Based on the Matching Pursuit Method. Journal of Analytical Sciences, Methods and Instrumentation, 2012, 2, 149-155. 